\title{
Managerial Power and CEO Pay ${ }^{1}$
}

\author{
Robert F. Göx ${ }^{2} \quad$ Thomas Hemmer ${ }^{3}$
}

April 2017, this draft February 2019

${ }^{1}$ The authors acknowledge helpful comments and suggestions from Davide Cianciaruso, Andrew Clausen, George Drymiotes (MAS discussant), Christian Laux, Thomas Pfeiffer (EIASM discussant), Ulrich Schäfer, Stefan Wielenberg (VFSP discussant), and seminar participants at the 2017 annual meeting of the VFSP accounting committee in Konstanz, the AAA's 2018 MAS midyear meeting, EAA 2018, EIASM Workshop on Accounting and Economics, HEC Paris, and UCLA.

${ }^{2}$ University of Zurich, Department of Business Administration, Chair of Managerial Accounting, Seilergraben 53, CH-8001 Zürich, Switzerland. Email: robert.goex@business.uzh.ch.

${ }^{3}$ Jones Graduate School of Business, Rice University, Houston TX 77005. Email: thomas.hemmer@rice.edu 


\title{
Managerial Power and CEO Pay
}

\begin{abstract}
We study how the CEO's power over the board of directors affects pay levels and the structure of optimal compensation contracts and derive unexpected results. First, a more powerful CEO generally receives more pay and a contract with a higher pay-performance sensitivity (PPS) if firm performance is low. In contrast, if firm performance is high, more CEO power translates into less pay and a lower PPS. Second, considering a special case of our general model, we show that more powerful CEOs receive higher salaries, more stocks but a nonincreasing number of options. Third, we find that the presence of a powerful CEO generally leaves the optimal use of relative performance evaluation unaffected. However, we identify conditions under which the sensitivity of CEO pay to peer performance can be increasing in the CEO's power over the board. Overall, our results suggest that frequently used indicators of poor (or sound) compensation practices should be interpreted with care.
\end{abstract}

Keywords: executive compensation, optimal contracting, managerial power theory.

JEL Classification: J33, M40 


\section{Introduction}

The question of whether or not the executive compensation practice in public firms reflects arm's length bargaining between managers and shareholders or rent seeking of the CEO is one of the most fundamental and controversial issues in empirical compensation research (Bebchuk and Grinstein 2005, Frydman and Jenter 2010, Core and Guay 2010). The "arms length bargaining perspective" suggests that executive compensation contracts are designed by boards acting in the best interest of shareholders in order to mitigate the agency problem caused by the separation of ownership and control.

In contrast, the "managerial power perspective" argues that weak governance structures allow CEOs to exercise power over the board of directors (subsequently also simply board) and thereby to control the level and structure of their own pay. According to Bebchuk and Fried (2004), weak boards tend to inflate CEOs' pay levels at the cost of shareholders by proposing inefficient compensation contracts featuring an insufficient link between pay and firm performance and/or a lack of control for common risk factors in measuring firm performance. Thus, ceteris paribus, the compensation arrangements implemented by managerial power firms should exhibit one ore more of the following characteristics: the compensation is generally too high, the pay-performance sensitivity is too low, the contract exhibits reward for luck, or, equivalently, a lack of relative performance evaluation (RPE). ${ }^{1}$

Despite the importance of this fundamental issue and the intensity of the academic debate initiated by Bebchuk and Fried (2004), there is surprisingly little theoretical research that addresses the question of whether and how optimal compensation contracts design by CEOfriendly boards differ from those designed to serve the best interest of the shareholders. ${ }^{2}$ In this paper, we aim to close this gap by providing a general framework for the analysis of the contracting problem faced by CEO-friendly boards. To that end, we modify the

\footnotetext{
${ }^{1}$ The managerial power approach was put forward by Bebchuk and Fried (2004). Bertrand and Mullainathan (2001) provide theoretical arguments and empirical evidence for presence of reward for luck in executive pay. Core et al. (2005) and Weisbach (2007) provide critical discussion of the managerial power approach and its key hypotheses.

${ }^{2}$ In a recent survey of the theoretical research on executive pay, Edmans and Gabaix (2016) note that a theoretical model of the managerial power hypothesis could be particularly useful as a benchmark for empirical compensation research because currently, the managerial power approach is mainly based on verbal arguments.
} 
standard agency model proposed by Mirrlees (1974, 1999) and Holmström (1979) in two ways. First, instead of as in the basic formulation having the residual claimant(s) (the "principal") design the contract with CEO (or "agent") this task in our model is delegated to a board of directors. The rationale for this is that the principal here is taken to be an everchanging group of anonymous and unrelated shareholders lacking the means to coordinate directly.

The board is an integral part of the firm as required by law and specified in by-laws. However, depending on its structure and the individuals serving on the board, the board can exhibit varying degrees of preference alignment with shareholders and the CEO. We capture this in our model by assuming that the board maximizes a weighted average of the principal's and the agent's utility. Second, we recognize formally that both shareholders and the manager have outside opportunities. Accordingly, our model exhibits participation constraints for both the CEO and for the firm's shareholders to assure that the contracts proposed by the board are accepted by both parties regardless of which of the parties the board favors. ${ }^{3}$ To ensure tractability of our analysis of the consequences of these changes to the standard model, we rely on the conditions proposed by Jewitt (1988) to guarantee that the first-order approach remains valid.

The specific analysis of our thus augmented principal-agent model suggests that while more management-friendly boards affect both the pay level and the structure of the optimal compensation contract, the implications of having a management friendly board are much more subtle than what is generally suggested by the managerial power approach. First, and importantly, we find that the structure of the optimal compensation contract only varies with the preferences of the board if neither the agent's nor the principal's participation constraint is binding. In fact, we find that there is a lower range of moderately CEO-friendly boards, i.e., board that actually put a moderately positive weight on the CEO's utility but still propose the same compensation contract as a board acting in the best interest of shareholders.

At the other end of the spectrum, there is an upper range of CEO-friendly board types that put a large weight on the CEO's utility and propose the same contract that the CEO

\footnotetext{
${ }^{3}$ It is standard to assign all the bargaining power to either the principal (for example Holmström 1979) or the manager (for example Jensen and Meckling 1976) which makes the participation constraint for the thus advantaged party redundant.
} 
would offer himself if he was in charge of the compensation decision. This contract is set so that the shareholders' participation constraint is binding and all rents thus are allocated to the CEO here. Finally, we identify an intermediate range of board types that write contracts so that no participation constraint is binding and both parties receive rents. It is only in this intermediate range, that the contract structure varies with the preferences of the board. At the limits of this range, the optimal contract coincides with the optimal contracts implemented in the lower and the upper range, respectively.

The optimal compensation contract in our model has several interesting properties. First, the compensation level is not simply an increasing function of the degree to which the board is friendly to the CEO. We find that there exists a critical performance level below which the compensation level is increasing as the board puts a higher weight on the CEO's utility and decreasing if the firm performance exceeds this critical level. Second, we find that the pay-performance sensitivity (PPS) depends on the curvature of the compensation function and the firm's realized performance. The PPS is only constant if the optimal compensation contract is linear in the likelihood ratio and the likelihood ratio is a linear function of the underlying performance measure. Third, while there is always a range of performance levels for which the contract provided by a CEO-friendly board exhibits a lower PPS, we identify conditions under which the PPS is increasing as the board puts a higher weight on the CEO's utility. Only if the optimal contract is linear in the firm's performance, it always exhibits a lower PPS than a contract designed in the best interest of shareholders.

To gain further insights on the influence of the board's preference on the optimal structure of the compensation contract, we analyze an optimal quadratic compensation contract in the spirit of Hemmer, Kim, and Verrecchia (2000). The optimal contract in this setting is comprised of a mix of cash- and equity-based compensation that can readily be interpreted as a salary, stock grants, and a convex component that approximates the payoff profile of stock options and thus closely resembles the mix of cash-, stock-, and option-based incentives found in public firms. Using this interpretation we find that a more friendly board pays a higher salary and uses more stock but a lower or an unchanged number of options to incentivize the CEO.

Interestingly, the optimal number of options and the aggregate effect of these changes on the compensation function depend on the curvature of the agent's cost function. If the cost 
function is strictly convex, a more friendly board proposes a contract with less options but if the cost function is linear, the optimal number of options does not vary with the preferences of the board. Aggregating these effects, we find that in the former case, the optimal contract provides the agent with more pay and a higher PPS for low performance levels but with lower pay and a higher PPS for high performance levels. In contrast, if the agent's cost function is linear, a CEO-friendly board not only raises the CEO's overall compensation but also increases the PPS relative to the contract proposed by a shareholder-friendly board.

As an extension of the baseline model, we examine how a CEO-friendly board affects the optimal use and the aggregation of multiple performance measures in the agent's compensation contract. We find that the friendliness of the board neither affects the firm's decision on the performance measures used in the compensation contract nor the rules for aggregating multiple performance measures into a single performance index. However, extending the optimal quadratic contracting setting in section 5, we first demonstrate that the sensitivity of the CEO's pay to peer performance is a decreasing function of the firm's own performance and an increasing function of peer performance. Moreover, in the parameter range where the optimal contract structure is affected by the structure of the board, we identify conditions under which a more CEO-friendly board evaluates the CEO's performance even more intensively against the performance of its peers than a board acting in the best interest of shareholders.

Overall, these observations suggest that poor governance quality from the perspective of the shareholders need not go hand in hand with inflated pay levels, a low pay-performance sensitivity, reward for luck, or a lack of RPE. If high pay levels and a high PPS are associated with high levels of firm performance, both measures are most likely an indicator of sound compensation practices, whereas a high pay level and a high PPS in the presence of a low firm performance should be taken as an indicator for a compensation policy that primarily serves the interest of the CEO rather than the interest of shareholders. Moreover, since the sensitivity of the CEO's pay-to-peer-performance is a linear function of the firm's own PPS with the opposite sign, a higher PPS is always associated with a more negative weight on the performance of peer firms. Thus, a more intensive use of RPE is only an indicator for sound compensation policies if the same holds for the PPS. Particularly, in the quadratic contracting setting with a linear cost function, a higher PPS and a more intensive use of RPE are clear indicators of a management-friendly compensation policy. 
To explore the importance of our use of an optimal contracting model for deriving these results, we briefly study how the presence of a CEO-friendly board as here defined affects the insights provided by the popular LEN model that is frequently cited as a theoretical benchmark for an arm's length contract in empirical compensation studies. We show that the optimal incentive rate of a LEN model does not vary with the preference structure of the board. In that model, the only consequence of CEO power on the optimal linear contract is that the board allocates the surplus of the agency to the CEO whenever his utility is more important for the board than the utility of shareholders. Otherwise, the optimal contract is equivalent to the standard LEN contract written in the best interest of shareholders. Since these predictions can hardly be reconciled with the observed changes in compensation practices over the past three decades, this suggests using the LEN model as a theoretical benchmark for the empirical analysis of the relation between governance quality and compensation policy might be difficult to justify.

To the best of our knowledge, this paper provides the first theoretical analysis of the relation between the CEO-friendliness of a board and the level and structure of CEO compensation using Holmström's (1979) standard moral hazard model. This model and its calibrated version studied in Hemmer et al. (2000) are particularly convenient for analyzing the underlying research question for at least two reasons. First, these models impose no explicit restrictions on the structure of the optimal compensation contract and second, they deliver non-trivial predictions regarding the consequences of managerial power for the PPS because the agent's pay and the firm's performance are continuous functions and the CEO's compensation varies with his power over the board.

Other authors such as Laux and Mittendorf (2010) or Ferri and Göx (2018) study the optimal compensation policy of management-friendly boards in the context of agency models with a binary outcome and risk neutral agents protected by limited liability. Within reasonable limits of CEO power, the optimal contract in such a model takes a particularly simple form: It comprises a constant salary satisfying the agent's limited liability constraint and a bonus for good performance that is monotonically increasing in the CEO's power over the board. While the second result is consistent with the predictions of Bebchuk and Fried (2004), the model provides only limited insights into the consequences of CEO power for the optimal structure of compensation contracts and the relation between CEO pay and firm performance due to its binary outcome structure. 
Dikolli et al. (2018) examine the impact of CEO power on the use of relative performance evaluation using a LEN model. Different from our model, the authors of this study assume that the agent's salary and the rewards for the firm's own performance are set in line with the interest of the firm's shareholders but that the CEO can decide on the contract weight that the firm puts on the performance of its peer. Using these assumptions, the authors find that the optimal linear compensation contract removes less common risk from the agent's compensation than a contract where all parameters are designed by the firm's shareholders. This result stands in sharp contrast with the predictions of our general model in section 6 , where the board's choice set is not restricted to single contract parameter and the optimal contract is allowed to be nonlinear. ${ }^{4}$

The rest of this paper is organized as follows. In section 2, we lay out the model assumptions. In section 3, we derive the structure of the optimal compensation contract. In section 4 , we discuss important properties of the optimal contract and the impact of a management-friendly board on the PPS. In section 5, we provide further insights into the structure of the optimal compensation contract employing the quadratic contract structure studied in Hemmer et al. (2000). In section 6, we study how the presence of a CEO-friendly board affects the optimal use and aggregation of multiple performance measure in the agent's compensation contract. In section 7, we discuss the suitability of the linear agency model for studying the relation between the power and pay of CEOs, and in section 8 we end this paper with a summary and some suggestions for future research.

\footnotetext{
${ }^{4}$ In line with our results in section 6 , it is easy to demonstrate that the power of the CEO has no impact on the optimal incentive rates in a LEN setting if the CEO or a management-friendly board are allowed to decide on all contract parameters.
} 


\section{Model}

We consider an agency problem as in Holmström (1979). A risk and effort-averse CEO (the agent) exerts unobservable effort $a \in[0, \bar{a}]$ on behalf of a group of risk neutral shareholders (the principal). The agent has an additively separable utility function $U(s)-C(a)$, where $U(s)$ is the utility derived from monetary compensation $s$ and $C(a)$ is the agent's personal cost of effort. We assume that $U_{s}>0, U_{s s}<0, C_{a}>0$, and $C_{a a} \geq 0$, where subscripts denote (partial) derivatives. The agent's effort stochastically affects the firm's output $x \in[\underline{x}, \bar{x}]$ via the distribution $F(x, a)$ with twice continuously differentiable density function $f(x, a)$. Let

$$
\Phi(y, a)=\int_{\underline{x}}^{y} F(x, a) d x \quad \text { and } \quad h(x, a)=\frac{f_{a}(x, a)}{f(x, a)},
$$

we assume that

$$
\begin{aligned}
& \Phi_{a}(y, a) \leq 0, \quad \Phi_{a a}(y, a) \geq 0 \quad \forall \quad y, \\
& h_{x}(x, a)>0, \quad h_{x x}(x, a)=0 \quad \forall \quad a .
\end{aligned}
$$

Condition (2) states that the agents' effort induces second-order stochastic dominance for the output distribution with diminishing returns. ${ }^{5}$ Condition (3) requires that the likelihood ratio is linearly increasing in $x .^{6}$ To compensate the agent for his personal effort, the firm offers the agent a performance-based compensation contract $s(x)$, where (3) satisfies that $s$ is increasing in $x$. With these assumptions, the expected utilities of the principal (4) and the agent (5) are:

$$
\begin{aligned}
V(s, a) & =\int(x-s(x)) f(x, a) d x, \\
H(s, a) & =\int U(s(x)) f(x, a) d x-C(a) .
\end{aligned}
$$

Different from much of the previous literature, we assume that the decision on the agent's compensation contract is not taken by the firm's shareholders but by a board of directors

\footnotetext{
${ }^{5}$ That is, for any two effort levels $a_{0}$ and $a_{1}=a_{0}+k$, it holds for $k>0$ that $Z\left(a_{0}, k\right)=\Phi\left(y, a_{0}\right)-\Phi\left(y, a_{0}+k\right)$ is strictly positive and decreasing in $a_{0}$.

${ }^{6}$ The class of probability distributions with linear likelihood ratios has been widely used in the agency literature (e.g. Amershi and Hughes 1989, Banker and Datar 1989). It includes, among others, the exponential distribution, the gamma distribution, the (censored) normal distribution, and the lognormal distribution.
} 
that maximizes a weighted average of the agent's and the principal's utilities in (4) and (5)

$$
B(s, a)=(1-\gamma) \cdot V(s, a)+\gamma \cdot H(s, a)
$$

Following extant literature (e.g. Drymiotes 2007 or Kumar and Sivaramakrishnan 2008), the weighting factor $\gamma \in[0,1]$ measures the power of the CEO over the board or, equivalently, the board's friendliness towards the CEO. The higher $\gamma$, the more weight the board puts on the CEO's utility in deciding on $s(x)$. At the extremes, if $\gamma=1$, the board's objectives are perfectly aligned with those of the CEO, whereas for $\gamma=0$, the board sets the CEO's compensation as to maximize shareholder value. In the following, we study how the friendliness of the board towards the CEO (subsequently also the board's affinity with the CEO or simply the board's type) affects the structure and the level of the CEO's compensation.

The board's problem consists of maximizing (6) subject to the following three constraints

$$
\begin{aligned}
H_{a}(s, a) & =\int U(s(x)) \cdot f_{a}(x, a) d x-C_{a}(a)=0 \\
H(s, a) & \geq \underline{H} \\
V(s, a) & \geq \underline{V}
\end{aligned}
$$

Conditions (7) and (8) are the agent's incentive and participation constraints, respectively. Condition (9) is the shareholders' participation constraint, where $\underline{H}$ and $\underline{V}$ are the parties respective reservation utilities. Unless stated otherwise, we subsequently normalize both to zero. Different from the standard agency model formulation in accounting where the principal is assigned all bargaining power and the agent's participation constraint thus is binding, the current model must also consider a participation constraint for shareholders. This constraint is a standard assumption of many contracting models in the finance literature (e.g. Tirole 2006, chapter 3). It assures that shareholders receive at least the net return from alternative investment opportunities (i.e. $\underline{V}$ ) and prevents that an overly managementfriendly board implements an extreme compensation contract that discourages shareholders from funding the firm.

While the parties' reservation utilities are generally determined by market forces, the CEO's power over the board of directors represents a deviation from arm's length contracting in the sense of the managerial power approach. That is, holding the reservation utilities of the CEO and shareholders fixed, an increase of $\gamma$ measures the quality of the firm's corporate 
governance in terms of the board's willingness to deviate from the standard contract that assigns the residual surplus of the firm to its owners. Of course, changes of the CEO's reservation utility would affect the solution of the agency problem as much as changes of the shareholders' reservation utility in a model where the CEO had all bargaining power. However, since the resulting changes of the optimal compensation contract are the result of changing market forces in both cases, these changes cannot be interpreted as the result of governance failures. Thus, to study how the deviations from arm's length contracting in the sense of the managerial power approach affect the firm's compensation policy, market forces and governance variables must be represented by independent model parameters to arrive at meaningful predictions as it is the case in our model.

Following Jewitt (1988), the incentive compatibility constraint in (7) defines a unique solution for the agent's optimal effort choice if conditions (2) and (3) are met, the expected output is nondecreasing and concave in $a,{ }^{7}$ and the agent's utility derived from the optimal compensation contract,

$$
w(z)=U(S(z))
$$

is concave in its argument $z .^{8}$ To assure the validity of the first-order approach, we assume that these conditions are met. To avoid unnecessary case distinctions we let $w^{\prime \prime}(z)<0$ throughout the analysis of the general model in sections 3 and 4 and study the special case where $w(z)$ is linear in section 5 . In addition, we restrict our attention to the class of utility functions where $K(s)=1 / U_{s}(s)$ is concave and

$$
\omega(x)=w^{\prime}(z(x)) \cdot h(x, a)
$$

is increasing and concave in $x$ provided that the argument of the agent's compensation function $z(x)$ is increasing in $x .^{9}$

While the concavity of $K(s)$ restricts our analysis to the empirical relevant class of convex compensation contracts and rules out the unusual case of a strictly concave compensation function, the second condition implies that the agent's marginal utility and the likelihood

\footnotetext{
${ }^{7}$ As shown by Conlon (2009b), the concavity of expected output is equivalent to condition (2). See also Conlon (2009a) for a comprehensive discussion of the first-order approach to the principal agent problem.

${ }^{8}$ Considering that $S(z)=U_{s}^{-1}(1 / z)$, it must hold that $-U^{\prime \prime} / U^{\prime} \geq S^{\prime \prime} /\left(S^{\prime}\right)^{2}$ for $w(z)$ to be concave.

${ }^{9}$ Of course, $\omega^{\prime \prime}(x)<0$ only if $w^{\prime \prime}(x)<0$ and otherwise linear in $x$ because $w^{\prime}(z)$ is constant if $w^{\prime \prime}(x)=0$.
} 
ratio have a negative covariance. ${ }^{10}$ Put differently, high performance realizations are associated with a low marginal utility and vice versa. This assumption seems quite natural considering that the marginal utility is positive and decreasing in $x$ due to the concavity of $w(z(x))$ whereas the likelihood ratio is increasing and negative for $x<E[x]$.

Our model assumptions are not very limiting and satisfied by many utility functions and output distributions typically used in the agency literature. For example, they are met by well-known distributions such as the exponential, the gamma, or the Poisson distribution and a large subclass of the HARA (hyperbolic absolute risk aversion) utility function taking the form

$$
U(s)=\frac{(1-q)}{q} \cdot M(s)^{q}, \quad \text { where } \quad M(s)=\frac{A \cdot s}{1-q}+B>0,
$$

$A>0$, and $q \in[0,1 / 2]$. Considering the range of $q$, our model encompasses a wide range of utility functions such as power utility or logarithmic utility. In line with our model assumptions, this utility function has the following properties: $K(s)=A^{-1} \cdot M(s)^{1-q}$ is concave in $s$ if $q \in[0,1)$ which implies that the compensation function

$$
S(z)=(1-q) \cdot\left(A^{\frac{q}{1-q}} \cdot z^{\frac{1}{1-q}}-\frac{B}{A}\right)
$$

is increasing and convex in $z$ if $q \in[0,1)$. Substituting (13) back into the agent's utility function yields the utility derived from the convex compensation contract

$$
w(z)=U\left(K^{-1}(z)\right)=\left(\frac{1-q}{q}\right) \cdot A^{\frac{q}{1-q}} \cdot z^{\frac{q}{1-q}},
$$

which is increasing and concave in $z$ as $q \in[0,1 / 2]$. It is straightforward to verify that $\omega(x)=w^{\prime}(z(x)) \cdot h(x, a)$ is increasing and concave in $x$ if $z(x)$ and $h(x, a)$ are affine functions of $x$.

\footnotetext{
${ }^{10}$ While these condition keep the analysis of our model tractable, they are neither necessary nor sufficient for the existence of an optimal solution to the agency problem. Of course, $S(z)$ can also be concave in $z$, which is the case if $K(s)$ is convex in $s$. For example, if $U(s)=-\exp (-s), K(s)=\exp (s)$ is convex and $S(z)=\ln (z)$ is concave. See Hemmer et al. (2000) for a detailed discussion of the implication of the agent's utility function for the curvature of the optimal compensation contract.
} 


\section{Optimal contract with a friendly board}

In this section, we study how varying degrees of board-friendliness affect its compensation policy. Proposition 1 characterizes the structure of the optimal contract found after maximizing the board's objective function (6) subject to the agent's incentive constraint in (7), his participation constraint in (8), and the shareholders' participation constraint in (9). Let $\mu, \lambda$, and $\nu$ be the Lagrangian multipliers associated with the constraints in (7), (8), and (9), respectively. Then,

Proposition 1: For a given board type $\gamma$, the optimal compensation contract takes the form

$$
\frac{1}{U_{s}(s(x))}=\alpha+\beta \cdot h(x, a)
$$

where

$$
\alpha=\frac{\gamma+\lambda}{1-\gamma+\nu}, \beta=\frac{\mu}{1-\gamma+\nu} .
$$

The equilibrium value of the contract's performance sensitivity, $\beta$, equals

$$
R(a) \equiv-\frac{V_{a}}{H_{a a}}
$$

for all values of $\gamma, \mu$ and $\nu$, where $R(a)$ is an increasing function of a. Proof: See appendix.

As shown in the proof of Proposition 1, the optimal solution of the board's contracting problem is determined by the contract structure in (15), the parameter $\beta$ in (17), and the agent's incentive constraint regardless of wether or not one of the two participation constraints is binding. The incentive constraint in (7) is always binding and determines the agent's equilibrium effort for given contract parameters $\alpha$ and $\beta$. For a given effort level, the sensitivity parameter $\beta$ is defined by (17). This parameter determines the weight that the contract puts on the likelihood ratio and thereby the performance sensitivity of the optimal compensation contract.

Because $V(s, a)$ and $H(s, a)$ do not directly depend on $\gamma$, the sensitivity parameter $\beta=$ $R(a)$ only varies with the friendliness of the board if the agent's incentive constraint and thereby his effort choice is a function of $\gamma$. In contrast, the other contract parameter, $\alpha$, is determined by the relevant participation constraint and the friendliness of the board. There are three alternative solutions of the board's contracting problem we need to consider. First, the case where the agent's participation constraint is binding, second, the case where the 
shareholders' participation constraint is binding and finally a third scenario, where none of the two participation constraints are binding. Corollary 1 establishes the condition where the agent's participation constraint is binding and summarizes the properties of the optimal contract for this case.

Corollary 1: If the board has a moderate affinity with the $C E O$, so that $\gamma \in[0, \underline{\gamma}]$, where $\underline{\gamma}>0$, it designs the contract so that the agent's incentive and participation constraints are binding. The parameters of the optimal compensation contract $\underline{s}$ in (15) take the form

$$
\underline{\alpha}=\alpha(\underline{a}), \quad \underline{\beta}=R(\underline{a}),
$$

where $\underline{\alpha}$ solves the agent's participation constraint and $\underline{a}$ the optimal effort level induced by the contract $\underline{s}$. The critical value of $\gamma$ up to which this solution is optimal, equals

$$
\underline{\gamma}=\frac{\underline{\alpha}}{1+\underline{\alpha}}
$$

and the Lagrangian multipliers take the values

$$
\nu=0, \quad \lambda(\gamma)=\underline{\alpha}-(1+\underline{\alpha}) \cdot \gamma, \text { and } \quad \mu(\gamma)=(1-\gamma) \cdot \underline{\beta}
$$

Proof: Follows from Proposition 1 considering $\nu=0$ and $\lambda>0$.

The optimal contract in (15) with the parameters given in (18) replicates the solution of the standard agency model in Holmström (1979). In Holmström's model, the board's objectives are perfectly aligned with those of shareholders $(\gamma=0)$ and there is no participation constraint for the firm's shareholders $(\nu=0)$. It is easy to see that for $\gamma=0$, the parameters of the compensation contract in (18) are identical to the Lagrangian multipliers in (20), i.e. $\lambda(0)=\underline{\alpha}$ and $\mu(0)=\underline{\beta}$, so that the contract takes the well-known form

$$
\frac{1}{U_{s}(s(x))}=\lambda+\mu \cdot h(x, a)
$$

Interestingly, the contract in (21) not only maximizes the utility of a board acting in the best interest of shareholders but also the utility of boards exhibiting a moderate affinity with the CEO. In fact, all boards with types $\gamma \leq \underline{\gamma}$ offer the same contract to the CEO. The optimal contract maximizes shareholder value as well as the utility of a moderately management-friendly board whenever it designs the contract so that the agent's incentive 
and participation constraints are binding. According to equation (16), the board sets the contract parameters and Lagrangian multipliers so that following identities are satisfied

$$
\underline{\alpha}=\frac{\gamma+\lambda(\gamma)}{1-\gamma} \text { and } \quad \underline{\beta}=\frac{\mu(\gamma)}{1-\gamma}
$$

These identities uniquely determine the values of the Lagrangian multipliers as long as the agent's participation constraint (8) is binding.

As can be seen from (20), the optimal values of both Lagrangian multipliers are decreasing in $\gamma$ because a higher affinity with the CEO shifts the board's objectives away from the interest of shareholders and thereby reduces the board's opportunity cost of meeting the agent's participation and incentive constraints. However, as long as $\lambda(\gamma)$ is nonnegative, this fact does not affect the optimal contract parameters and the agent's effort choice because the identity in (22) assures that an increase of $\gamma$ is balanced by a corresponding decrease of $\lambda$ without changing $\underline{\alpha}$. This solution is optimal for the board whenever $\gamma \leq \underline{\gamma}$. For larger values of $\gamma, \lambda(\gamma)$ becomes negative so that it is no longer optimal for the board to fix $\alpha$ so that (8) is binding.

On the other hand, if the board becomes so friendly towards the CEO that $\gamma>\underline{\gamma}$, it optimally designs the contract so that the shareholders' participation constraint is binding $(\nu>0)$ but the agent's participation constraint has slack $(\lambda=0)$. This contract allocates the net surplus of the agency to the CEO and leaves shareholders with the expected value of their outside investment opportunity. However, this extreme solution of the agency problem is optimal only if the board's affinity with the CEO exceeds an upper bound $\bar{\gamma}$.

Corollary 2: If the board has a strong affinity with the CEO, so that $\gamma \in[\bar{\gamma}, 1]$, it designs the contract so that the agent's incentive constraint and the shareholders' participation constraint are binding. The parameters of the optimal compensation contract $\bar{s}$ take the form

$$
\bar{\alpha}=\alpha(\bar{a}), \quad \bar{\beta}=R(\bar{a})
$$

where $\bar{\alpha}$ solves the shareholders' participation constraint, $R(a)$ is defined in (17) and $\bar{a}$ is the optimal effort level induced by the contract $\bar{s}$. The critical value of $\gamma$ above which this solution is optimal, equals

$$
\bar{\gamma}=\frac{\bar{\alpha}}{1+\bar{\alpha}}
$$

The Lagrangian multipliers take the values

$$
\lambda=0, \quad \nu(\gamma)=\frac{\gamma}{\bar{\gamma}}-1, \text { and } \quad \mu(\gamma)=\gamma \cdot \frac{\bar{\beta}}{\bar{\alpha}} .
$$


Proof: Follows from Proposition 1 considering $\nu>0$ and $\lambda=0$.

As for the case of moderately friendly boards in Corollary 1, the optimal contract in (23) takes the same form for a range of possible board types above a critical value of $\bar{\gamma}$. The upper bound of this range is represented by a board that maximizes the utility of the CEO but puts zero weight on the interest of shareholders $(\gamma=1)$. For this extreme case, the Lagrangian multipliers in (25) take the values $\nu(1)=1 / \bar{\alpha}$ and $\mu(1)=\bar{\beta} / \bar{\alpha}$. For other values of $\gamma \in[\bar{\gamma}, 1]$, the values of the Lagrangian multipliers are defined by the identities

$$
\bar{\alpha}=\frac{\gamma}{1-\gamma+\nu(\gamma)} \quad \text { and } \quad \bar{\beta}=\frac{\mu(\gamma)}{1-\gamma+\nu(\gamma)}
$$

Similar to the result in Corollary 1, these identities uniquely determine the values of the Lagrangian multipliers as long as the shareholders' participation constraint (9) is binding. Different from the previous case, both Lagrangian multipliers are increasing in $\gamma$ indicating that an increasing affinity with the CEO raises the board's opportunity cost of satisfying the shareholders' participation constraint and the agent's incentive constraint. ${ }^{11}$ This solution is optimal for the board whenever $\gamma \geq \bar{\gamma}$. For lower values of $\gamma, \nu(\gamma)$ becomes negative and the board no longer benefits from fixing the value of $\alpha$ so it solves (9).

Finally, there is a third solution to the board's contracting problem where it designs the contract so that none of the two participation constraints is binding:

Corollary 3: Whenever the board has an intermediate affinity with the CEO, where $\gamma \in$ $(\underline{\gamma}, \bar{\gamma})$, it designs the contract so that only the agent's incentive constraint $(7)$ is binding. For this case, the parameters of the optimal contract are

$$
\alpha(\gamma)=\frac{\gamma}{1-\gamma}, \quad \beta(\gamma)=R(a(\gamma))
$$

where $a(\gamma)$ denotes the agent's equilibrium effort for a given affinity $\gamma$. Within the intermediate range, the Lagrangian multipliers take the values

$$
\nu=\lambda=0 \text {, and } \quad \mu=(1-\gamma) \cdot \beta(\gamma)
$$

Proof: Follows from Proposition 1 considering $\nu=\lambda=0$.

\footnotetext{
${ }^{11}$ While the first effect is quite intuitive, the second effect stems from the fact that $\mu$ is decreasing in $\gamma$ and increasing in $\nu$. Since $\nu$ is increasing in $\gamma$ and this indirect effect is stronger than the direct effect, $\mu$ is increasing in $\gamma$.
} 
Unlike in the standard agency model formulation where the principal has been preassigned all bargaining power and designs the contract so that the agent receives just his reservation utility, a friendly board does not necessarily transfer the total surplus to the CEO. Instead, the board can find it optimal to propose a contract that splits the surplus of the agency between the CEO and the firm's shareholders so that the expected utility of both parties exceeds their reservation utility. To see why this policy is optimal for boards with affinity levels $\gamma \in(\underline{\gamma}, \bar{\gamma})$, it is helpful to compare the properties of the board's objective function for the three solutions in Corollary 1, 2, and 3.

Suppose that the board proposes the contract in Corollary 1. Since the contract allocates the surplus of the agency to shareholders and the agent's participation constraint is binding, it must hold that

$$
V(\underline{a}, \underline{s})>0 \text { and } H(\underline{a}, \underline{s})=0
$$

By contrast, if the board implements the contract in Corollary 2, the players' utilities equal

$$
V(\bar{a}, \bar{s})=0 \text { and } H(\bar{a}, \bar{s})>0 .
$$

Substituting the expressions in (28) and (29) into the board's objective function yields $\underline{B}(\gamma)=(1-\gamma) \cdot V(\underline{a}, \underline{s})$ in the former case and $\bar{B}(\gamma)=\gamma \cdot H(\bar{a}, \bar{s})$ in the latter case. Evidently, both functions are linear in $\gamma$ but since a more friendly board puts a lower weight on the principal's utility and a higher weight on the agent's utility, $\underline{B}(\gamma)$ is decreasing in $\gamma$, whereas $\bar{B}(\gamma)$ is increasing in $\gamma$. Thus, there exists a level of friendliness beyond which the board prefers the contract in Corollary 2 over the standard contract in Corollary $1 .^{12}$

Let $\Gamma(\gamma)$ denote the board's objective function evaluated at the optimal contract and equilibrium effort defined in Corollary 3. This function is strictly convex in $\gamma$, decreasing in $\gamma$ for values of $\gamma<\gamma^{*}$, increasing in $\gamma$ for values of $\gamma>\gamma^{*}$, and $\gamma^{*}$ is the value of $\gamma$ where $\Gamma(\gamma)$ takes its minimum. Since $\underline{B}(\gamma)$ and $\bar{B}(\gamma)$ are linear and $\Gamma(\gamma)$ is strictly convex in $\gamma$, but it holds that $\Gamma(\underline{\gamma})=\underline{B}(\underline{\gamma})$ and $\Gamma(\bar{\gamma})=\bar{B}(\bar{\gamma})$, it must be that $\Gamma(\gamma)>\max \{\underline{B}(\gamma), \bar{B}(\gamma)\}$ if $\gamma \in(\underline{\gamma}, \bar{\gamma})$. Quite intuitively, the convexity of $\Gamma(\gamma)$ follows from the fact that $H(\cdot)$ is monotonically increasing in $\alpha, V(\cdot)$ is monotonically decreasing in $\alpha$. Because $\alpha$ is increasing in $\gamma$ and the board puts more weight on $H(\cdot)$ but less weight on $\alpha$ as $\gamma$ gets larger, it must be that $\Gamma(\gamma)$ has an U-shaped curvature.

\footnotetext{
${ }^{12}$ In fact, it is easy to see that $\bar{B}(\gamma) \gtreqless \underline{B}(\gamma)$ if $\gamma \gtreqless \frac{H(\bar{a}, \bar{s})}{H(\bar{a}, \bar{s})+V(\underline{a}, \underline{s})}$.
} 
[insert Figure 1 about here]

Figure 1 illustrates how the optimal compensation contracts summarized in Corollaries 1-3 affect the board's objective function for different values of $\gamma$ using a numerical example. The dashed downward sloping line represents $\underline{B}(\gamma)=(1-\gamma) \cdot V(\underline{a}, \underline{s})$, the dotted upward sloping line represents $\bar{B}(\gamma)=\gamma \cdot H(\bar{a}, \bar{s})$, and the solid U-shaped curve represents $\Gamma(\gamma)$. It can be seen that the unconstrained objective function $\Gamma(\gamma)$ lies above the expected utilities resulting from the two constrained solutions except for the tangency points $\gamma=\underline{\gamma}$ and $\gamma=\bar{\gamma}$ where the unconstrained solution yields the same expected utility as the relevant constrained solution.

Of course, the board could attain a higher utility level if it could also implement the unconstrained solution for $\gamma<\underline{\gamma}$. However, such a solution would violate the agent's participation constraint because it requires that the board sets the contract parameter $\alpha$ so that $\alpha(\gamma)=\gamma /(1-\gamma)<\underline{\alpha}$. Likewise, the unconstrained solution violates the shareholders' participation constraint if $\gamma>\bar{\gamma}$ because in this case, the board must set $\alpha$ so that $\alpha(\gamma)>\bar{\alpha}$ to implement the unconstrained solution. Thus, the unconstrained solution can only be implemented if $\gamma \in(\underline{\gamma}, \bar{\gamma})$ where it yields a strictly higher expected utility than any of the two constrained solutions.

Clearly, the existence of the solution in Corollary 3 requires that $\underline{\gamma}<\bar{\gamma}$. In the proof of Lemma 1, we show that this condition is always met under the assumptions of our model. In addition, we establish the following result:

Lemma 1: Provided the three solutions to the board's contracting problem in Corollaries 1-3 exist, the agent's effort level is decreasing in $\gamma$. Proof: See appendix.

According to Corollary 3, a more friendly board implements a lower equilibrium effort than a board acting in the interest of a shareholders. The intuition behind this result can be explained as follows. As we have shown in Proposition 1, a change of $\gamma$ can only affect the agent's effort choice via the agent's incentive constraint because $H(\cdot)$ and $V(\cdot)$ do not directly depend on $\gamma$. Since $\gamma$ can only enter the agent's compensation contract via $\alpha$, and $\alpha$ is increasing in $\gamma$, the result in Lemma 1 can best be understood by studying how a change of the parameter $\alpha$ affects the agent's effort choice. Let

$$
U(\alpha, a, y)=\int_{0}^{y} u(\alpha, a, x) d x
$$


denote the distribution of the agent's utility derived from monetary compensation where the density of this distribution,

$$
u(\alpha, a, x)=w(\alpha+\beta \cdot h(x, a)) \cdot f(x, a),
$$

is the product of the output density and the utility function in (10) as a function of the optimal contract structure in (15). This distribution defines the agent's expected monetary benefits from choosing productive effort $a$. Because a higher value of $\alpha$ raises the agent's compensation independent of the firm's performance level, an increase of $\alpha$ shifts the distribution induced by $a$ to the left. That is, for a given effort level $a$ and arbitrary contract parameters $\alpha_{2}>\alpha_{1}$ it holds that $U\left(\alpha_{2}, a, y\right)>U\left(\alpha_{1}, a, y\right)$ for all output levels $y \in[\underline{x}, \bar{x}]$. As we show in the proof of Lemma 2, this shift to the left reduces the agent's marginal utility for a given effort level and thereby causes him to choose a lower effort level as the board becomes more friendly.

\section{Properties of the optimal compensation contract}

In this section, we study how the friendliness of the board affects the agent's compensation and the pay-performance sensitivity (PPS). Lemma 2 summarizes some essential properties of the optimal compensation function:

Lemma 2: Let $s(x, \gamma)=S(z(x, \gamma))$ denote the agent's compensation function for the contract structure defined in Corollary 3, where $S(z)=U_{s}^{-1}(1 / z)$ as defined in (10),

$$
z(x, \gamma)=\alpha(\gamma)+\beta(a(\gamma)) \cdot h(x, a(\gamma))
$$

and $\gamma \in(\underline{\gamma}, \bar{\gamma})$. The following holds:

1. $S(z)$ is monotonically increasing in $z$ and $z(x, \gamma)$ is monotonically increasing in $x$.

2. $\alpha(\gamma)$ is monotonically increasing in $\gamma$ and $\beta(\gamma)$ is decreasing in $\gamma$.

Proof: See appendix.

First, for any type of increasing and concave utility function $U(s)$, the agent's compensation function $S(z)$ is monotonically increasing in its argument $z(x, \gamma)$ which by itself is an 
affine function of the likelihood ratio $h(x, a)$. Because we restrict our analysis to the class of probability distributions where the likelihood ratio is linear in $x$, the argument of the agent's compensation function is linearly increasing in the firm's performance. In contrast, the optimal compensation function is strictly convex in $x$ if $K(s)=1 / U_{s}(s)$ is strictly concave and only linear in $x$ if $K(s)$ is linear. For example, considering the HARA utility function in (12), the optimal compensation function in (13) is linear in $x$ if $q=0$ and convex in $x$ if $q \in(0,1 / 2]$.

Second, we find that a friendly board determines the argument of the agent's compensation function so that it raises the constant $\alpha(\gamma)$ but puts a lower weight $\beta(\gamma)$ on the likelihood ratio. While the first effect is obvious from the definition of $\alpha(\gamma)$ in $(27)$, the second effect follows from combining the facts that $\beta=R(a)$ is increasing in the agent's effort but that $a(\gamma)$ is decreasing in $\gamma$. Proposition 2 summarizes how the friendliness of the board affects the agent's compensation level and the PPS:

Proposition 2: Let $\pi(x, a)=R(a) \cdot h(x, a)$ denote the performance-based component in $z$, let $P(x, \gamma)=s_{x}(x, \gamma)$ denote the pay-performance sensitivity of the optimal compensation contract, and suppose that $\gamma \in(\underline{\gamma}, \bar{\gamma})$. We make the following observations:

1. There exists a critical performance level $x_{0}$ so that the agent's compensation $s(x, \gamma)$ is increasing in $\gamma$ if $x \leq x_{0}$ and decreasing in $\gamma$ if $x>x_{0}$.

2. $P(x, \gamma)$ is nondecreasing in $x . P(x, \gamma)$ is constant only if $S(z)$ is linear.

3. There is a range of performance levels for which $P(x, \gamma)$ is decreasing in $\gamma$.

(a) If $S(z)$ is linear, $P(x, \gamma)$ is decreasing in $\gamma$ for all $x \in[\underline{x}, \bar{x}]$.

(b) If $S(z)$ is strictly convex, there exists a critical performance level $x_{1} \in[\underline{x}, \bar{x})$ so that $P(x, \gamma)$ is decreasing in $\gamma$ if $x>x_{1}$ but increasing in $\gamma$ if $x \leq x_{1}$.

Proof: See appendix.

The first key result in Proposition 2 is the observation that a friendly board not necessarily pays a higher compensation to the CEO. In fact, we identify quite general conditions under which a friendly board finds it optimal to raise the CEO's compensation for low levels of firm performance but to cut the CEO's compensation for high levels of firm performance. 
As we show in the proof of the proposition, there is always a cut-off level $x_{0}$ beyond which a friendly board lowers the CEO's compensation relative to a board acting in the interest of shareholders. Thus, according to our analysis, high pay-levels in the presence of a high firm performance are an indicator of a compensation policy in the best interest of shareholders, whereas high pay levels in the presence of low firm performance indicate a compensation policy that favors the CEO.

The second insight in Proposition 2 is the fact that the magnitude of the PPS is usually not a constant but determined by the curvature of the optimal compensation function and the realized performance level $x$. Clearly, the slope of the compensation function must vary with the realized performance level whenever the compensation function is not linear in $x$. Since the slope of a strictly convex function is increasing in its argument, it must be that the PPS is increasing if the compensation function if $S(z)$ is strictly convex. Thus, even for the class of probability distributions where the likelihood ratio is linear in $x$, the PPS is only independent of the realized firm performance if the optimal contract is linear in $x$.

The third key insight provided by Proposition 2 is the observation that a friendly board not necessarily provides the CEO with a contract that exhibits a lower PPS. Interestingly, we find that the PPS is only generally decreasing in $\gamma$ if the optimal compensation contract is indeed linear in $x .{ }^{13}$ More generally, we find that for the wide class of strictly convex optimal compensation contracts where the agent's effort varies with the friendliness of the board, the PPS can in fact be decreasing or increasing in $\gamma$. To be precise, we identify conditions for the existence of a cut-off level $x_{1}$ below which the optimal contract provided by a friendly board exhibits a higher PPS than the contract proposed by a board acting in the best interest of shareholders.

Taken together, these results clearly contradict the implicit suppositions of the managerial power approach that poor governance quality goes hand in hand with inflated pay levels and a weaker link between pay and firm performance. According to our analysis, the impact of friendly boards on CEO pay levels and the PPS cannot be determined independent of the firm's performance level due to the convexity of the optimal compensation contract. For the

\footnotetext{
${ }^{13}$ This result contradicts the predictions of the widely used LEN model where the linearity of the compensation contract is imposed by a convenient set of model assumptions but not derived as the optimal solution of the boards' contracting problem. We elaborate this point in more detail in section 7
} 
same reason, the magnitude of the PPS cannot be taken as an indicator for the severeness of the underlying agency problem without relating it to the realized performance level. In the next section, we will explore these issues in greater detail in the context of a quadratic setting that allows us to derive the optimal compensation contract in closed form.

\section{An optimal quadratic compensation contract}

To gain further insights into the consequences of an imperfect preference alignment between the board and shareholders for the firm's compensation policy, we study the properties of an optimal convex compensation contract that exhibits the main features of equity-based incentive schemes used by most public firms. Suppose that the agent has power utility $U(s)=q^{-1} \cdot s(x)^{q}$ with $q=1 / 2$ which is a special case of the HARA utility function in (12) considering that $A=1 / 2$ and $B=3 s$. In line with Hemmer et al. (2000), we let the firm performance follow a gamma distribution with density function

$$
f(x, a)=\frac{\left(\frac{x}{a}\right)^{k-1} \cdot e^{-\frac{x}{a}}}{a \Gamma(k)},
$$

where $k$ is a nonnegative scaling parameter. The parameter affects both the shape of the density function and the moments of the distribution. For low values of $k$, the density is skewed to the left, whereas for higher values of $k$, the density tends to become more centered around the mean. With $E[x]=k a$ and $V A R[x]=k a^{2}$, the expectation and the variance of $x$ are increasing in $k$. In line with our general model, the likelihood ratio of the gamma distribution is linear in $x$ and takes the form

$$
h(x, a)=\frac{x-E[x]}{a^{2}} .
$$

It is easy to see that the likelihood ratio is increasing in $x$, decreasing in $a$ for $x>E[x] / 2$, and that its slope is decreasing in the agent's effort, i.e. $h_{x a}<0$. With these assumptions, the compensation contract takes the quadratic form $s(x)=z(x)^{2}$. Using the definition of $z(x)$ in $(32)$ and defining

$$
s_{0}(\alpha, a)=\alpha-k \cdot \frac{\beta(a)}{a}, \quad s_{1}(a)=\frac{\beta(a)}{a^{2}}
$$

the compensation function can be decomposed into three additive components so that

$$
s(x)=w+n \cdot x+m \cdot x^{2},
$$


where $w=s_{0}(\alpha, a)^{2}, n=2 \cdot s_{0}(\alpha, a) \cdot s_{1}(a)$, and $m=s_{1}(a)^{2}$. Suppose that $x$ represents the firm's stock price, then we can interpret $w$ as the salary, $n$ as the number of stocks, and the quadratic term in (34) adds a convex component that approximates the typical payoff structure provided by stock options (Core et al. 2003). ${ }^{14}$ For this contract structure, the PPS takes the form

$$
P(x)=n+2 m x,
$$

which is an affine function of the firm's stock price. The constant of this function is determined by the size of the stock-based component and the slope is determined by the size of the option-like component. The magnitude of all three components of the compensation contract depend on the agent's equilibrium effort and the optimal values of the contract parameters $\alpha$ and $\beta$ chosen by the board. ${ }^{15}$ Proposition 3 summarizes how the contract components vary with the magnitude of the CEO's power over the board.

Proposition 3: In the quadratic contract setting, the salary and the slope of the linear component $(n)$, are increasing in $\gamma$. The slope of the convex component $(m)$ is nonincreasing in $\gamma$. It is decreasing in $\gamma$ if $C^{\prime \prime}(a)>0$ and constant if $C^{\prime \prime}(a)=0$. Proof: see appendix.

According to Proposition 3, the friendliness of the board affects the three components of the optimal quadratic compensation contract in a different manner. While the salary and the magnitude of the stock-based compensation component are increasing as the board becomes more friendly, the magnitude of the option component is (weakly) decreasing as the board's preferences become sufficiently aligned with the preferences of the CEO. The intuition for these results is straightforward. Since the agent's marginal utility is an increasing function of $\beta$ and the incentive constraint requires that the marginal utility equals the marginal cost of effort, $\beta(a)=a^{2} \cdot C^{\prime}(a) \cdot(2 k)^{-1}$ is increasing in $a$ and $C^{\prime}(a)$. Using this relation allows us

${ }^{14}$ As Larcker and Tayan (2011) point out, the convex payout profile from stock options is often even accelerated by premium options that provide a a leveraged payout profile for high performance levels.

${ }^{15}$ The key advantage of the quadratic contract is the separate identifiability of equity-based pay components. For $q \in[0,1 / 2)$ the optimal contract still satisfies the conditions of our general model but takes the form $s(x)=\left(s_{0}+s_{1} \cdot x\right)^{\frac{1}{1-q}}$, where $s_{0}$ and $s_{1}$ are defined in (33). For this contract, the optimal effort level and the corresponding contract parameters can no longer be determined in closed form but the salary and the aggregate equity-based component of this contract can be readily identified as $W=s_{0}^{\frac{1}{(1-q)}}$ and $M=s(x)-W$. However, taking $x$ as the stock price, $M$ now represents the total amount of equity-based pay that can no longer be split up into a stock and an option-based component. 
to eliminate $\beta(a)$ from the structural parameters of the compensation contract in (33) and to rewrite these expressions as

$$
s_{0}(\alpha, a)=\alpha-\frac{a \cdot C^{\prime}(a)}{2}, \quad s_{1}(a)=\frac{C^{\prime}(a)}{2 k} .
$$

Of course, $w$ is increasing in $s_{0}(\cdot)$ and $s_{0}(\cdot)$ is increasing in $\alpha$ and decreasing in $a$. Because $\alpha$ is increasing in $\gamma$ and $a$ is decreasing in $\gamma$, both effects work into the same direction so that the salary must be increasing in $\gamma$. This effect does not vary with the curvature of the agent's cost function because $\alpha$ is independent of $C(a)$ and $a \cdot C^{\prime}(a)$ is increasing in $a$ even if $C(a)$ is linear. In contrast, $s_{1}(a)$ is only an increasing function of $a$ if $C(a)$ is strictly convex but independent of $a$ if $C(a)$ is linear. Considering that $m$ is increasing in $s_{1}(a)$ and $a$ is decreasing in $\gamma$, the option component must be decreasing in $\gamma$ in the former case and decreasing in $\gamma$ in the latter case. Finally, since $n$ is increasing in $s_{0}(\cdot)$ and $s_{1}(\cdot)$, it is obvious that the stock component is increasing in $\gamma$ if $C(a)$ is linear. If $C(a)$ is strictly convex, the net effect of a more friendly board on the amount of stock grants is generally ambiguous because $s_{0}$ is increasing and $s_{1}$ is decreasing in $\gamma$. However, as we show in the proof of Proposition 3, in equilibrium the former effect always dominates the latter effect so that a more friendly board always grants more stock to the CEO.

Corollary 4: In the quadratic contract setting, a more friendly board provides the CEO with a contract that exhibits a higher pay level and a higher PPS for all $x$ whenever $C(a)$ is linear in a. If $C(a)$ is strictly convex, the contract has the properties of the general convex contract in Proposition 2. Proof: Follows from Proposition 3.

Corollary 4 underscores the importance of the agent's cost function for studying the impact of a friendly board on the curvature of the optimal compensation function and the PPS. Perhaps most surprisingly, if $C(a)$ is linear in $a$, a more friendly board not only offers the agent a higher compensation for all levels of firm performance but also raises the optimal PPS relative to the contract offered by a shareholder-friendly board. Considering the definitions of the optimal contract in (34) and the PPS in (35), this result follows quite intuitively from that fact that $w$ and $n$ are increasing in $\gamma$ whereas $m$ is constant.

In contrast, if $C(a)$ is strictly convex, the optimal contract has the same properties as the general contract in Proposition 2. That is, a more friendly board increases the agent's pay and the PPS for low performance levels and decreases both measures for high performance levels. These effects are both caused by the fact that a more friendly board increases the 
salary and the stock component but cuts back the option component if the cost function is strictly convex. Clearly, since $s(0)=w$ and $P(0)=n$, the compensation and the PPS for low performance levels must be higher than those offered by a board acting in best interest of shareholders. However, since the option component is lower but its effect on $s(x)$ and $P(x)$ is increasing in $x$, there are critical performance levels above which the pay level and the PPS are lower than those offered by a shareholder-friendly board.

[insert Figure 2 about here]

Figure 2 illustrates how the friendliness of the board and the curvature of the cost function jointly determine the shape of the compensation function and the pay-performance sensitivity. Panel A shows both functions for the strictly convex cost function $C(a)=a^{2}$, whereas Panel B shows the corresponding results assuming the linear cost function $C(a)=a$. In all figures, we compare the optimal compensation functions and the PPS for the limiting cases of the compensation contracts in Corollaries 1 and 2. The dashed lines show the curvature of the standard contract where $\gamma=\underline{\gamma}$ and the agent's participation constraint is binding. The solid lines show the curvature of the contract where $\gamma=\bar{\gamma}$ and the shareholder's participation constraint is binding.

It can bee seen that the solid line in the left figure of Panel A lies above the dashed line for performance levels below $x_{0}$ and above it for performance levels beyond $x_{0}$. Thus, in line with Proposition 2, a friendly board provides the CEO with higher pay for moderate performance levels and with lower pay for high performance levels relative to a board acting in the best interest of shareholders whenever the cost function is strictly convex. The left figure of Panel B shows the curvature of the two compensation contracts for a linear cost function. It can be seen that the solid curve lies everywhere above the dashed curve indicating that a CEOfriendly board raises the CEO's compensation for all performance levels if the cost function is linear.

The two figures at the right of Panels A and B compare the pay-performance sensitivity for the two compensation contracts in Corollaries 1 and 2. In both figures, the PPS is an increasing function of $x$. As before, the dashed lines show the PPS of the standard contract, whereas the solid lines shows the PPS of the contract where the shareholders' participation constraint is binding. As for the left figure of Panel A, the solid line lies above the dashed 
line for low performance levels if $x<x_{1}$ and above it if $x>x_{1}$. Even though $x_{1}<x_{0}$, there is a lower range of performance levels for which the contract proposed by a friendly board exhibits a higher PPS than the contract proposed by a board acting in the best interest of shareholders. In contrast, the solid line in the right figure of Panel B lies everywhere above the dashed line indicating that a CEO-friendly board not only raises the CEO's pay level but also the PPS for all performance levels if the cost function is linear.

In summary, these observations underscore the fact that a poor governance quality need not go hand in hand with inflated pay levels and a low pay-performance sensitivity. On one hand, the quadratic contract combined with a linear cost function illustrates that a management-friendly board can find it optimal to propose a more generous compensation contract that exhibits a higher sensitivity to firm performance. On the other hand and in line with the results of the general model, the results of the quadratic contract for a strictly convex cost function suggests that pay levels and the PPS should be considered relative to the firm's realized performance when evaluating the board's compensation policy. If high pay levels and a high PPS are associated with high performance, both measures are more likely an indicator of sound compensation practices. In contrast, a high pay level and a high PPS in the presence of a low performance should be taken as an indicator for a compensation policy that primarily serves the interest of the CEO rather than the interest of shareholders.

\section{Friendly boards and the lack of RPE}

In this section, we study how the presence of a management-friendly board affects the use and aggregation of additional performance measures in addition to firm performance $x$. This aspect of the problem is of particular interest because several proponents of the managerial power approach appear to view weak governance structures as the main reason for the insufficient use of relevant performance measures in CEO compensation contracts. As a consequence, they maintain that the presence of a management-friendly board could explain suboptimal compensation practices such as reward for luck (Bertrand and Mullainathan 2001) or the lack of relative performance evaluation in executive compensation (Bebchuk and Fried 2004).

To examine the validity of this argument, we extend the base model in section 3 by 
introducing a second performance signal $y$ where the joint distribution of $x$ and $y$ is $F(x, y, a)$ with twice continuously differentiable density function $f(x, y, a)$. Let

$$
h(x, y, a)=\frac{f_{a}(x, y, a)}{f(x, a)},
$$

denote the associated likelihood ratio. It follows directly from Proposition 1, that the optimal contract then takes the form

$$
\frac{1}{U_{s}(s(x, y))}=\alpha(\gamma)+\beta(\gamma) \cdot h(x, y, a),
$$

where $\alpha(\gamma)$ and $\beta(\gamma)$ are defined in (16). The structure of the expression in (38) implies the following observation:

Proposition 4: A contract s(x,y) can only improve the solution implemented by the optimal contract $s(x)$ if it is false that

$$
f(x, y, a)=g(x, y) \cdot z(x, a)
$$

The degree of the board's affinity with the CEO does not affect the firms' decision on the use of $y$. Proof: the first part is shown in Holmström (1979), the second part follows from the fact that the informativeness criterion in (39) does not dependent on $\gamma$.

Proposition 4 implies that the question of wether or not firms use additional performance measures in optimal compensation contracts only depends on their informativeness in the presence of $x$ but it is not affected by the CEO's power over the board. We next examine whether and how the board's affinity with the CEO affects the optimal aggregation of performance measures in the optimal contract. As shown by Banker and Datar (1989), the optimal aggregation rule is linear if the joint density function belongs to the class

$$
f(x, y, a)=\exp \left[z_{1}(a) \cdot y+z_{2}(a) \cdot x-z_{3}(a)+z_{4}(y)+z_{5}(x-\rho \cdot y)\right]
$$

where $z_{i}$ are arbitrary functions of $a, x$ and $y$.

Proposition 5: Suppose that the joint density function belongs to the class in (40), then the optimal contract can be decomposed into a compensation function $s(z(x, y))$, and an aggregate performance measure $z(x, y)$ that is linear in $x$ and $y$ and takes the form

$$
z(x, y)=z_{1 a}(a) \cdot y+z_{2 a}(a) \cdot x
$$


The relative weights on $x$ and $y$ in the aggregate performance measure are only affected by the boards affinity with the CEO if the ratio

$$
r(x, y)=h_{x}(x, y, a) \cdot h_{y}(x, y, a)^{-1}
$$

varies with the agent's effort. Proof: see appendix

According to Proposition 5, the presence of managerial power can only affect the wellknown rules for the optimal linear aggregation of performance measures if none of the two participation constraints is binding. This condition is equivalent to the result for the simple contract $s(x)$ in Corollary 3. However, different from the findings in section 3, this requirement is not sufficient to affect the optimal aggregation of the two performance signals in the agent's compensation contract. In addition, it must be that the ratio of marginal changes of the likelihood ratio with respect to the changes of the two performance measures depends in a non-trivial way on the agent's effort. As shown in the appendix, the optimal aggregation rule is independent of the board's affinity with the CEO whenever the marginal likelihood ratios take the form $h_{i}(x, y, a)=k_{i} \cdot l(a)$ for $i=x, y$ because in this case $r(x, y)$ is a constant.

However, even if the board's friendliness towards the CEO does not affect the optimal linear aggregation of performance measures, the optimal weight that the board puts on the aggregate performance index in the optimal compensation contract will typically vary with $\gamma$. To illustrate this point, suppose that the joint density function takes the form of a bivariate gamma distribution

$$
f(x, y, a)=\frac{\left(\frac{x \cdot y-b \cdot y^{2}}{a \cdot \kappa}\right)^{k-1} \cdot e^{-\left(\frac{\kappa \cdot x+(a-\kappa \cdot b) \cdot y}{\kappa \cdot a}\right)}}{a \cdot \kappa \cdot \Gamma(k)^{2}},
$$

where $b$ and $\kappa$ are arbitrary positive constants. This class is a special case of the exponential class in (40) where only $x$ depends on the agent's effort but $y$ is an informative signal in the sense of Holmström (1979). With the density in (43), it is straightforward to calculate the expectations of $x$ and $y$ yielding $E[x]=a \cdot k+b \cdot E[y]$ and $E[y]=\kappa \cdot k$. Thus, if we interpret $y$ as the stock price of a peer firm or a given peer index, expected firm performance is positively affected by the agent's effort and the performance of its peers.

Taking the derivative with respect to $a$, the likelihood ratio becomes

$$
h(x, y, a)=\frac{x-b \cdot y-a \cdot k}{a^{2}} .
$$


As before, $h(x, y, a)$ is an affine function of the underlying performance measures. Applying the aggregation rule in (41), the optimal contract takes the same form as in (15) except for fact that $x$ is replaced by the aggregate performance index ${ }^{16}$

$$
v(x, y)=x-b \cdot y
$$

Since $b=C O V(x, y) \cdot V A R(y)^{-1}$, the aggregate performance measure in (45) can be interpreted as a net measure of firm performance after eliminating the impact of $y$ measured in a linear regression of firm performance on the performance of its peers.

Using the notation for the arguments in the compensation contract in (33), the general version of the contract in Proposition 2 takes the form $s(x, y)=S\left(s_{0}(\alpha, a)+s_{1}(a) \cdot v(x, y)\right)$ and the sensitivities of the agent's pay to its own firm performance and the performance of its peer are:

$$
P_{x}(x, y)=s_{1}(a) \cdot S^{\prime}\left(s_{0}(\alpha, a)+s_{1}(a) \cdot v(x, y)\right) \quad \text { and } \quad P_{y}(x, y)=-b P_{x}(x, y)
$$

In line with the results in section 5, the expressions in (46) indicate that the sensitivity of the agent's pay with respect to his own performance is not a constant but an increasing function of the aggregate performance index $v(x, y)$, whereas the sensitivity of the agent's pay with respect to peer performance is decreasing in $v(x, y)$. Since $v(x, y)$ is increasing in $x$, decreasing in $y$, and the same observation holds for $P_{x}(x, y)$, it follows that $P_{y}(x, y)$ is decreasing in $x$ and increasing $y$. Thus, higher realizations of the corresponding performance variables have a positive impact on the sensitivities of compensation with respect to these measures.

Because $s(x, y)$ is a special case of the general contract in Proposition 2, all previous results regarding the relation between the firm's own PPS and the friendliness of the board continue to hold, albeit the statements must consider changes in the aggregate performance index $v(x, y)$ instead of considering changes of $x$ only. Moreover, since the pay-to-peerperformance is a linear function of the firm's own PPS with the opposite sign, a higher PPS

\footnotetext{
${ }^{16}$ Of course, the use of the aggregate performance measure in (45) requires that the contract is bounded from below for high realizations of $y$ to assure that the right hand side of the optimality condition in (15) is always positive. However, as shown by Holmström (1977, chapter 4) and noted by Banker and Datar (1989), a bounded sharing does not change the structure of the optimal compensation rule for the interior region of performance levels for which the right hand side of (15) is positive.
} 
implies a lower pay-to-peer-performance sensitivity. Thus, for the performance regions where the firm's own PPS is increasing in $\gamma$, the pay-to-peer-performance sensitivity is decreasing in $\gamma$ and vice versa. This observation implies that a more friendly board can either evaluate the CEO more or less intensively relative to peer performance, where a lower (i.e. more negative) weight on $y$ is equivalent to a more intensive use of RPE. That is, whenever a more friendly board proposes a contract with a higher PPS, it also makes more intensive use of RPE such as in the quadratic contracting setting with a linear cost function studied in section 5 .

These results clearly do not align well with the standard argument of the managerial power approach according to which a powerful CEO always forces the board to reward him for positive market trends. Overall, our results suggest that the relation between the firm's governance structure and the optimal aggregation of additional performance measures are by far more subtle than conventional wisdom might suggest.

\section{Friendly boards in a LEN setting}

In the light of our results, it is interesting to see in how far the insights provided by the general model can also be derived from the analysis of a LEN model. This question is of particular interest for empirical compensation research because the optimal LEN contract is frequently used as the theoretical benchmark for an arm's length compensation contract despite the conceptual difficulties associated with its use. ${ }^{17}$ Since the model is simple and its results are well known, we will focus on the question of wether and how a friendly board affects the optimal contract derived from a LEN model.

In the LEN model, the firm performance is a linear function of the agent's effort and a noise term $\varepsilon \sim N\left(0, \sigma^{2}\right)$, the agent has negative exponential utility with constant absolute risk aversion $r$, and the compensation contract is linear in $x$. With $x=a+\varepsilon, s(x)=w+o \cdot x$ and a reservation utility of zero, the parameters of the linear compensation contract in the

\footnotetext{
${ }^{17}$ Among the widely cited empirical studies that refer to the LEN model as a theoretical benchmark for an optimal compensation contract are Jensen and Murphy (1990), Murphy (1999), and Murphy (2013). See Hemmer (2004) for a detailed discussion of the conceptual difficulties associated with the use of the LEN model in empirical compensation research.
} 
standard LEN model are

$$
\underline{o}=\frac{1}{1+r C^{\prime \prime}(\underline{a}) \sigma^{2}}, \quad \underline{w}=C(\underline{a})+\frac{r}{2} \underline{o}^{2} \sigma^{2}-\underline{o a} .
$$

At the other extreme, for a contract that maximizes the CEO's utility subject to the shareholders' participation constraint, the optimal contract parameters are

$$
\bar{o}=\frac{1}{1+r C^{\prime \prime}(\bar{a}) \sigma^{2}}, \quad \bar{w}=(1-\bar{o}) \bar{a}
$$

Comparing the solutions in (47) and (48) shows that even the most extreme shift in the preferences of the board only affects the size of the fixed payment but not the incentive rate of the optimal linear compensation contract. Consequently, both contracts must induce the same effort level $\underline{a}=\bar{a}$. In both contracts, the incentive rate is set so that it maximizes the total surplus of the agency,

$$
T C E=E[x]-C(a)-\frac{r}{2} o^{2} \sigma^{2}
$$

regardless of the player that is allowed to collect it. In a second step, the maximized surplus is divided between the principal and the agent according to the players' bargaining power by an appropriate calibration of the fixed payment. In the standard contract, the shareholders have all bargaining power and determine $w$ so that the agent's expected compensation equals the sum of his personal cost and his risk premium. In the second contract, the CEO has all bargaining power and calibrates the fixed payment so that his total compensation equals the surplus of the agency.

Proposition 6: In a LEN model, the optimal incentive rate is independent of $\gamma$. If $\gamma<1 / 2$, the optimal fixed payment is $\underline{w}$, if $\gamma>1 / 2$, the optimal fixed payment equals $\bar{w}$, and if $\gamma=1 / 2$, the board is indifferent between the two contracts. Proof: see appendix.

According to Proposition 6, the objective function of the board has no influence on the choice of the incentive rate in the CEO's compensation contract. The preferences of the board only affect the choice of the fixed payment. ${ }^{18}$ Whenever the board puts more weight on the utility of shareholders than on the utility of the CEO, it uses the fixed payment to transfer the entire surplus of the agency to the firm's shareholders. Likewise, if the board

\footnotetext{
${ }^{18}$ This result can only change if lump sum wealth transfers between the shareholders and the CEO are restricted or costly. See Göx (2008) for the analysis of an optimal LEN-contract with costly wealth transfers.
} 
puts more weight on the utility of the CEO than on the utility of shareholders, it calibrates the fixed payment so that the entire surplus is allocated to the CEO.

The reason for this result is simple. Unlike the general model, there is no solution for which none of the two participation constraints is binding. As a consequence, the board's objective function is always an affine transformation of the total surplus. This surplus is maximized by the incentive rate $o=\underline{o}=\bar{o}$, and the fixed payment just serves the purpose of transferring the maximized surplus to the player that dominates the preferences of the board. Thus, according to the predictions of the LEN model, we should not expect the preferences of the board to have a non-trivial impact on the structure of the optimal compensation contract other than an inflation of CEO salaries. These predictions cannot be reconciled with the evidence on the increasing significance of equity-based compensation components in CEOs' compensation packages over the past thirty years. As a consequence, using LEN-model as a theoretical benchmark for the empirical analysis of the relation between governance quality and compensation policy in public firms might be difficult to justify.

\section{Summary and conclusions}

The so called managerial power approach is frequently used as a theoretical basis for explaining the use of seemingly inefficient compensation practices such as inflated pay levels, a low pay-performance sensitivity, or the lack of RPE. In this paper, we provide a rigorous theoretical analysis of the arguments put forward by Bebchuk and Fried (2004) and other proponents of the managerial power approach. Our study is based on a modified version of the standard agency model, where we replace the principal by a board that maximizes a weighted average of the principal's and the agent's utility and add a participation constraint for the principal. To focus on the consequences of these changes on the predictions of the standard model, we limit our analysis to a setting where the first-order approach is valid.

Our analysis shows that the presence of a management-friendly board affects the structure of the optimal compensation contract and the agent's pay level only if the agent's participation constraint is not binding. In fact, we identify a range of moderately managementfriendly board types that propose the same compensation contract as a board acting in the best interest of shareholders. At the other extreme, there is an upper range of highly 
management-friendly boards that maximize the CEO's rent by proposing a contract where the shareholder's participation constraint is binding. Finally, there is an intermediate range of board types that seek a compromise between the interests of both players and implement a contract where none of the two participation constraint is binding. It is only in this intermediate range, that the structure of the optimal compensation contract varies with the preferences of the board.

The optimal compensation contract in our model has several properties that can hardly be reconciled with the simple hypotheses put forward by the managerial power approach. First, we find that the CEO's compensation level generally only increases in his power over the board if the firm performance falls below a critical threshold. For performance levels beyond this threshold, a more management-friendly board provides the CEO with lower pay. Thus, in the general version of our model, managerial power typically translates into higher pay for low performance levels but into lower pay for high performance levels. Likewise, we find that the pay-performance sensitivity (PPS) typically varies with the firm's performance. In line with our findings for the optimal pay level, we identify conditions where the contract offered by a management-friendly board exhibits a higher PPS than the standard contract written in the best interest of shareholders.

To provide some additional insights on the potential impact of the CEO's power on the optimal structure of CEO compensation, we study the properties of an optimal quadratic contract that closely resembles the mix of cash- and equity-based incentives found in public firms. This setting offers the advantage of identifying separate components of CEO pay that can readily be interpreted as a salary, a stock-based component, and a convex component that approximates the payoff profile of stock options. Using this interpretation, we find that a more management-friendly board pay a higher salary, grants more stock but offers the same or a lower number of options to incentivize the CEO. Aggregating these effects, we find that the optimal contract proposed by a friendly boards exhibits both a higher pay and a higher PPS as compared to the arm's length contract whenever the option component remains constant. Otherwise, the contract has the same properties as in the general model.

We also study an extension of the baseline model, where we examine how a friendly board affects the optimal use of multiple performance measures and their aggregation into a single performance index. First, we find that the degree of the board's alignment with shareholder 
interests has no consequences for the firm's choice of measures for evaluating the agent's performance. Second, we find that the CEO's power over the board generally does not affect the well-known standard results for the optimal linear aggregation of performance measures. However, we also identify conditions under which a more CEO-friendly board evaluates the CEO's performance even more intensively against the performance of its peers than a board acting in the best interest of shareholders.

Taken together, these observations suggest that each of the measures of seemingly flawed compensation practices proposed by the proponents of the managerial power approach is at best imperfect because it can equally indicate arm's length contracting. That is, high pay levels, a low pay-performance sensitivity, or a low intensity of RPE can reflect the rational choice of boards acting in the best interest of shareholders rather than the compensation policy of boards serving the interest of the CEO. Moreover, our analysis suggests that an accurate interpretation of these indicators requires a careful consideration of the subtle relation between the frequently used measures of the contractual efficiency, the curvature of the optimal contract, and the realized values of the underlying performance measures. 


\section{Appendix}

\section{Proof of Proposition 1}

The board maximizes its objective function in (6) subject to the constraints in (7), (8), (9). This optimization problem can be expressed by the Lagrangian

$$
L=(1-\gamma) \cdot V(s, a)+\gamma \cdot H(s, a)+\mu \cdot H_{a}(s, a)+\lambda \cdot H(s, a)+\nu \cdot V(s, a) .
$$

Pointwise differentiation of (49) with respect to $s$ yields the first-order condition

$$
L_{s}=(1-\gamma+\nu) \cdot V_{s}+(\gamma+\lambda) \cdot H_{s}+\mu \cdot H_{a s}=0
$$

where $V_{s}=-f(x, a), H_{s}=U_{s}(s(x)) \cdot f(x, a), H_{a s}=U_{s}(s(x)) \cdot f_{a}(x, a)$. Using these expressions and rearranging terms yields the results stated in equations (15) and (16). This condition defines the structure of the optimal compensation contract.

Given the optimal contract structure, the board's maximization problem generally has the three solutions summarized in Corollaries 1, 2, and 3. These solutions are determined by the condition

$$
L_{a}=(1-\gamma+\nu) \cdot V_{a}+\mu \cdot H_{a a}=0
$$

the agent's IC constraint, and the relevant participation constraint, provided that one of the two constraints binding. Here, the incentive constraint determines the equilibrium effort $a$ for given values of $\alpha$ and $\beta$. The optimal value of $\alpha$ is determined by the relevant participation constraint or, if no participation constraint is binding, by the value of $\gamma$. Finally, the optimal value of $\beta$ is determined by (51). Rearranging terms in (51) and comparing the result with (16) yields the expression in (17).

To see that $\beta=R(a)$ is increasing in $a$, consider how a change of $\beta$ affects the agent's equilibrium effort. Using the definition in (10), we can write the agent's utility for arbitrary contract parameters $\alpha$ and $\beta$ as

$$
w(z(x))=w(\alpha+\beta \cdot h(x, a)) .
$$

Using this definition and the fact that

$$
H_{a \beta}=\int w^{\prime}(z(x)) \cdot h(x, a) \cdot f_{a}(x, a) d x=\int w^{\prime}(z(x)) \cdot h(x, a)^{2} \cdot f(x, a) \cdot d x>0
$$


because $E\left[w^{\prime}(z(x)) \cdot h(x, a)^{2}\right]>0$, it follows from the implicit function theorem, that the agent's equilibrium effort is increasing in $\beta$ because $d_{a} / d_{\beta}=-H_{a \beta} / H_{a a}$ and $H_{a a}<0$. Thus, it holds that $\beta_{a}=R_{a}>0$.

\section{Proof of Lemma 1}

\section{Part 1: Friendly boards and equilibrium effort.}

Proposition 1 implies that the agent's equilibrium effort can only be affected by $\gamma$ if none of the participation constraints is binding. In this case, $\alpha$ is an increasing function of $\gamma$ by Corollary 3. To see how a change of $\alpha$ affects the agent's effort choice, we proceed as in the proof of Proposition 1 and examine how $H_{a}$ varies with $\alpha$. From the implicit function theorem $a^{\prime}(\alpha)$ has the same sign as

$$
H_{a \alpha}=\int w^{\prime}(z(x)) \cdot f_{a}(x, a) d x=\int w^{\prime}(z(x)) \cdot h(x, a) \cdot f(x, a) \cdot d x .
$$

Since $\omega(x)=w^{\prime}(z(x)) \cdot h(x, a)$ is increasing and strictly concave in $x$, and $E[h(E[x], a)]=$ $h(E[x], a)=0$ by the linearity of the likelihood ratio, Jensen's inequality implies that

$$
0=\omega(E[x])>E(\omega(x))
$$

it follows that $a^{\prime}(\alpha)=H_{a \alpha}<0$. Finally, since $\alpha$ is increasing in $\gamma$ from Corollary 3, we can readily conclude that

$$
\frac{d a}{d \gamma}=\frac{d a}{d \alpha} \cdot \frac{d \alpha}{d \gamma}<0
$$

\section{Part 2: Existence of the solution in (27).}

Recall first that $\underline{\alpha}$ solves the agent's participation constraint, whereas $\bar{\alpha}$ solves the shareholders' participation constraint. In the relevant equilibrium, these conditions take the form

$$
\begin{aligned}
& H(s(\underline{\alpha}, \underline{a}), \underline{a})=E[U(s(\underline{\alpha}, \underline{a}))]-C(\underline{a})=0, \\
& V(s(\bar{\alpha}, \bar{a}), \bar{a})=E[x(\bar{a})]-E[s(\bar{\alpha}, \bar{a})]=0 .
\end{aligned}
$$

Here, $s(\alpha, a)=s(\alpha, \beta(a), a, x)$ denotes the optimal compensation contract in (15). Since (55) is binding if $\gamma \leq \underline{\gamma}$ and (56) is binding if $\gamma \geq \bar{\gamma}$, the solution in (27) can only exist if $\bar{\gamma}>\underline{\gamma}$, or, equivalently if

$$
\underline{\alpha}<\alpha(\gamma)<\bar{\alpha}
$$


Thus, a sufficient condition for existence is that $\underline{\alpha}<\bar{\alpha}$. To see that there are always conditions for which (57) is satisfied, note that unconstrained objective functions of the agent and the principal satisfy the conditions

$$
\begin{aligned}
H_{\alpha}(s(\alpha, a), a) & =E_{\alpha}[U(s(\underline{\alpha}, \underline{a}))]>0 \\
V_{\alpha}(s(\alpha, a), a) & =V_{a} \cdot \frac{d a}{d \alpha}-E_{\alpha}[s(\bar{\alpha}, \bar{a})]<0
\end{aligned}
$$

because $V_{a}=-H_{a a} \cdot \beta(a)>0$, the agent's effort is decreasing in $\alpha$ and the expected compensation and the agent's expected utility from compensation are both increasing in $\alpha$. Further, since only one participation constraint can be binding it also holds that

$$
\begin{aligned}
& V(s(\underline{\alpha}, \underline{a}), \underline{a})>V(s(\bar{\alpha}, \bar{a}), \bar{a}), \\
& H(s(\bar{\alpha}, \bar{a}), \bar{a})>H(s(\underline{\alpha}, \underline{a}), \underline{a}) .
\end{aligned}
$$

Suppose now that (57) is violated so that $\underline{\alpha}>\bar{\alpha}$ and the the solution in (27) does not exist. With this assumption, (60) and $(61)$ can only be satisfied if $H_{\alpha}(\cdot)<0$ and $V_{\alpha}(\cdot)<0$, a contradiction to (58) and (59).

\section{Proof of Lemma 2}

1. To verify that $S(z)$ is monotonically increasing in $z$, it suffices to note that $K(s)=$ $1 / U^{\prime}(s)=z$ so that

$$
S^{\prime}(z)=\frac{1}{K^{\prime}(s)}=-\frac{U^{\prime}(s)^{2}}{U^{\prime \prime}(s)}>0
$$

because the agent's utility is concave in $s$. Likewise, $z_{x}=\beta(a) \cdot h_{x}(x, a)>0$ because $\beta(a)>0$ and $h_{x}(x, a)>0$.

2. The first part is obvious from the definition of $\alpha(\gamma)$ in $(27)$. To show that $\beta(\gamma)$ is decreasing in $\gamma$, we note first that

$$
\frac{d \beta(\gamma)}{d \gamma}=\frac{d \beta(a)}{d a} \cdot \frac{d a}{d \gamma}<0
$$

Since we have established in Proposition 1 and Lemma 1 that $\beta=R(a)$ is increasing in $a$ and that $a$ is decreasing in $\gamma$, it follows that $\beta$ is decreasing in $\gamma$. 


\section{Proof of Proposition 2}

To verify our claims, we first establish how $\pi(x, a)=\beta(a) \cdot h(x, a)$ varies with $x$ and $a$. To this end, we rewrite the likelihood ratio as an arbitrary affine function of $x$ so that $h(x, a)=$ $g_{1}(a) \cdot x-g_{2}(a)$, where $g_{1}(a)$ and $g_{2}(a)$ are arbitrary positive functions of $a$. Using the fact that $E[h(x, a)]=0$, we can rewrite the slope of $z(x)$ as

$$
\pi(x, a)=\beta(a) \cdot g_{1}(a)(x-E[x(a)])=p(a)(x-E[x(a)]),
$$

where $E[x(a)]=g_{2}(a) / g_{1}(a)$. It follows that

$$
\begin{aligned}
& \pi_{x}(x, a)=p(a), \quad \pi_{x a}(x, a)=p^{\prime}(a) \\
& \pi_{a}(x, a)=p^{\prime}(a)(x-E[x(a)])-p(a) \frac{d E[x(a)]}{d a}
\end{aligned}
$$

To see how $p(a)$ varies with the agent's effort, we rewrite the agent's utility as

$$
w(z(x))=w(\alpha+p(a)(x-E[x(a)])) .
$$

Using this expression and the fact that $p(a)$ is a constant in equilibrium, we totally differentiate the agent's incentive constraint and find that $d p(a) / d a=-H_{a a} / H_{a p}$, where

$$
H_{a p}=\int w^{\prime}(z(x)) \cdot \frac{h(x, a)^{2}}{g_{1}(a)} \cdot f(x, a) \cdot d x=\frac{H_{a \beta}}{g_{1}(a)}>0 .
$$

Because $H_{a a}<0$, we conclude that $d p(a) / d a>0$. Using the properties of $\pi(x, a)$, we proceed to verify the claims made in the Proposition.

1. To see how $s(x, \gamma)$ varies with $\gamma$, note first that

$$
\frac{\partial s}{\partial \gamma}=S_{z} \cdot \frac{d z}{d \gamma}
$$

where $S_{z}>0$ from Lemma 2 and

$$
\frac{d z}{d \gamma}=\frac{\partial \alpha}{\partial \gamma}+\pi_{a}(x, a) \cdot \frac{\partial a}{\partial \gamma}
$$

Thus, the agent's compensation can only be monotonically increasing in $\gamma$ if $d z / d \gamma>0$ for all $x$. Recall first that $\partial \alpha / \partial \gamma>0$ and $\partial a / \partial \gamma<0$ so that $d z / d \gamma>0$ if $\pi_{a}(x, a)<0$. However, as we can see from $(65), \pi_{a}(x, a)$ is negative if $x=E[x(a)]$ but monotonically increasing in $x$ and positive if

$$
x>E[x(a)]+\frac{p(a)}{p^{\prime}(a)} \frac{d E[x(a)]}{d a} .
$$


It follows that $d z / d \gamma>0$ if $x=E[x \mid a]$ but since $\partial \alpha / \partial \gamma$ is independent of $x$, there must be a critical performance level $x_{0}>E[x(a)]$ for which $\pi_{a}(x, a)$ is sufficiently large so that $d z / d \gamma<0$.

2. Let

$$
P(x, \gamma)=S_{z} \cdot \beta(a(\gamma)) \cdot h_{x}(x, a(\gamma))
$$

denote the pay-performance-sensitivity (PPS) of the optimal compensation contract. To prove the claim, note that

$$
\frac{\partial P(x, \gamma)}{\partial x}=S_{z z} \cdot\left[\left(\beta(a(\gamma)) \cdot h_{x}(x, a(\gamma))\right]^{2}+S_{z} \cdot \beta(a(\gamma)) \cdot h_{x x}(x, a(\gamma)) .\right.
$$

The sign of the first term in (69) depends on the curvature of the compensation function. Since $h(x, a)$ is linear in $x$, the second term is zero. It follows that the PPS is nondecreasing. That is, it is increasing in $x$ if $S(z)$ is strictly convex and constant if $S(z)$ is linear.

3. To determine how the PPS varies with the board's preferences, we differentiate $P(x, \gamma)$ with respect to $\gamma$ and obtain:

$$
\frac{\partial P(x, \gamma)}{\partial \gamma}=S_{z z} \cdot \frac{d z}{d \gamma} \cdot \pi_{x}(x, a)+S_{z} \cdot \pi_{x a}(x, a) \cdot \frac{\partial a}{\partial \gamma}
$$

a. If $S(z)$ is linear, equation (70) becomes

$$
\frac{\partial P(x, \gamma)}{\partial \gamma}=S_{z} \cdot \pi_{x a}(x, a) \cdot \frac{\partial a}{\partial \gamma}<0
$$

This expression is negative because $\pi_{x a}(x, a)=p^{\prime}(a)>0$ and $\partial a / \partial \gamma<0$.

b. If $S(z)$ is strictly convex, the first term in (70) is negative if $d z / d \gamma<0$ and positive if $d z / d \gamma>0$. Thus, the PPS is unambiguously decreasing in $\gamma$ if $x>x_{0}$ but increasing in $\gamma$ if $d z / d \gamma>0$ and the following condition is met

$$
S_{z z} \cdot \frac{d z}{d \gamma} \cdot \pi_{x}(x, a)>-S_{z} \cdot \pi_{x a}(x, a) \cdot \frac{\partial a}{\partial \gamma} .
$$

Provided that there exists a positive performance level $x_{1}>\underline{x}$ for which (71) holds, the PPS is increasing in $\gamma$ if $x<x_{1}$. Otherwise, the PPS is decreasing in $\gamma$ for all $x$. 


\section{Proof of Proposition 3}

Following the optimization program of the general model in section 3, the board maximizes the objective function

$$
B=(1-\gamma) \cdot\left(k \cdot a-\alpha^{2}-k \cdot \frac{\beta^{2}}{a^{2}}\right)+\gamma \cdot(2 \cdot \alpha-C(a))
$$

subject to the agent's incentive constraint,

$$
2 \cdot \beta \cdot \frac{k}{a^{2}}-C^{\prime}(a)=0
$$

and the two participation constraints (8) and (9). Since the contract parameters in (34) can only vary with $\gamma$ if none of the participation constraints is binding, it suffices to consider the case where $\lambda=\nu=0$. Using this fact and substituting for $\beta$ from the agent's incentive constraint yields the board's first-order condition for the optimal effort level

$$
k-\frac{a \cdot C^{\prime}(a) \cdot\left(C^{\prime}(a)+a \cdot C^{\prime \prime}(a)\right)}{2 \cdot k}-\alpha(\gamma) \cdot C^{\prime}(a)=0 .
$$

Since $\alpha(\gamma)=\gamma /(1-\gamma)$ is an increasing function of $\gamma$, a more friendly board puts a higher weight on the agent's cost function and implements a lower effort level. That is, in line with Lemma 1, the equilibrium effort $a(\gamma)$ is decreasing in $\gamma$.

To determine how the components of the optimal compensation contract vary with $\gamma$, let $s_{0}(\alpha(\gamma), a(\gamma))=s_{0}(\gamma)$, and $s_{1}(a(\gamma))=s_{1}(\gamma)$ and note that

$$
\begin{aligned}
& s_{0}^{\prime}(\gamma)=\frac{d \alpha}{\partial \gamma}-\frac{1}{2} \cdot\left[a \cdot C^{\prime \prime}(a)+C^{\prime}(a)\right] \cdot \frac{d a}{\partial \gamma}>0, \\
& s_{1}^{\prime}(\gamma)=\frac{C^{\prime \prime}(a)}{2 k} \cdot \frac{d a}{\partial \gamma} \leq 0,
\end{aligned}
$$

where $s_{1}^{\prime}(\gamma)=0$ if $C^{\prime \prime}(a)=0$ and $s_{1}^{\prime}(\gamma)<0$ if $C^{\prime \prime}(a)>0$. Using the definitions of the individual compensation components and the above results, it follows that

$$
\begin{aligned}
& \frac{d w}{d \gamma}=2 s_{0}(\gamma) \cdot s_{0}^{\prime}(\gamma)>0 \\
& \frac{d m}{d \gamma}=2 s_{1}(\gamma) \cdot s_{1}^{\prime}(\gamma) \leq 0 \\
& \frac{d n}{d \gamma}=2\left[s_{0}(\gamma) \cdot s_{1}^{\prime}(\gamma)+s_{1}(\gamma) \cdot s_{0}^{\prime}(\gamma)\right]>0
\end{aligned}
$$

where the last expression uses the fact that the stock based-component can be expressed as

$$
n(a)=1-\frac{1}{2}\left(\frac{1}{k^{2}}+\frac{1}{k}\right) \cdot a \cdot C^{\prime}(a)^{2}-\frac{1}{2 k^{2}} \cdot a^{2} \cdot C^{\prime}(a) \cdot C^{\prime \prime}(a)
$$


using the definitions of $s_{0}(\alpha, a)$ and $s_{1}(a)$ in (36) and substituting for $\alpha$ from (74). Since $n(a)$ is decreasing in $a$ and $a$ is decreasing in $\gamma$, it must be that

$$
\frac{d n(a(\gamma))}{d \gamma}=\frac{d n(a)}{d a} \cdot \frac{d a}{d \gamma}>0
$$

It follows that the salary and the stock-based component are monotonically increasing in $\gamma$, whereas the option component is constant if the cost function is linear and decreasing if the cost function is strictly convex.

\section{Proof of Proposition 5}

The first part of the statement follows directly from Banker and Datar (1989). The second part follows from the fact that the ratio does not directly depend $\gamma$ because we can see from the proof of Lemma 2 that

$$
\frac{s_{x}(x, y)}{s_{y}(x, y)}=\frac{-\frac{U_{s}^{2}}{U_{s s}} \cdot h_{x}(x, y, a)}{-\frac{U_{s}^{2}}{U_{s s}} \cdot h_{y}(x, y, a)}=\frac{z_{2 a}(a)}{z_{1 a}(a)} .
$$

It follows from Corollary 3, that the relative weights of the performance measures in (42) can only vary with $\gamma$ if $\gamma \in(\underline{\gamma}, \bar{\gamma})$ and if $z_{i a}(a) \neq k_{i} \cdot l(a)$.

\section{Proof of Proposition 6}

Let $C E P=E[x]-E[s(x)]$ and $C E A=E\left(s(x)-C(a)-\frac{r}{2} o^{2} \sigma^{2}\right.$ denote the certainty equivalents of the principal and the agent and consider the Lagrangian of the board's maximization problem

$$
L=(1-\gamma+\nu) \cdot C E P+(\gamma+\lambda) \cdot C E A+\mu \cdot\left(o-C^{\prime}(a)\right)
$$

Differentiating this expression with respect to $a, o$ and $w$ yields

$$
\begin{aligned}
L_{a} & =(1-\gamma+\nu) \cdot(1-o)+(\gamma+\lambda) \cdot\left(o-C^{\prime}(a)\right)-\mu \cdot C^{\prime \prime}(a)=0 \\
L_{o} & =(1-\gamma+\nu) \cdot a+(\gamma+\lambda) \cdot\left(a-o \cdot r \sigma^{2}\right)=0 \\
L_{w} & =2 \gamma-1+\lambda-\nu
\end{aligned}
$$

Suppose first, that the agent's participation constraint is binding so that $\nu=0, L_{w}=0$ and

$$
\lambda=1-2 \gamma
$$

Since $\lambda$ must be nonnegative if this solution is optimal, it is only feasible if $\gamma=\underline{\gamma} \leq 1 / 2$. 
Consider next the case where the shareholders' participation constraint is binding so that $\lambda=0, L_{w}=0$ and

$$
\nu=2 \gamma-1
$$

Since $\nu$ must be nonnegative if this solution is optimal, it is only feasible if $\gamma=\bar{\gamma} \geq 1 / 2$. Thus, since $\underline{\gamma}=\bar{\gamma}$, there is no value of $\gamma$ for which none of the two participation constraints

is binding. It follows that $w=\underline{w}$ if $\gamma<1 / 2$, and $w=\bar{w}$ if $\gamma>1 / 2$. If $\gamma=1 / 2$, the board is indifferent between the two contracts. To see that the optimal incentive rate is independent of $\gamma$, it suffices to solve (75) for $\mu$ and to substitute the optimal values of $\lambda$ and $\nu$ for the two cases into (76) using the fact that $o=C^{\prime}(a)$ from the agent's incentive constraint.

\section{References}

Amershi, A. H. and J. S. Hughes (1989): Multiple Signals, Statistical Sufficiency, and Pareto Orderings of Best Agency Contracts. The RAND Journal of Economics, 20(1), 102-112

Banker, R. D. and S. M. Datar (1989): Sensitivity, precision, and linear aggregation of signals for performance evaluation. Journal of Accounting Research, 27(1), 21-39.

Bebchuk, L. A. and J. M. Fried. 2004. Pay without Performance - The unfulfilled Promise of Executive Compensation. Cambridge, MA and London: Harvard University Press.

Bebchuk L. and Y. Grinstein (2005). The Growth of Executive Pay, Oxford Review of Economic Policy 21(2), 283-303.

Bertrand, M. and S. Mullainathan (2001): Are CEOs rewarded for Luck? The Ones without Principals are, The Quarterly Journal of Economics 116(3), 901-932.

Conlon, John R. (2009a): Two New Conditions Supporting the First-Order Approach to Multisignal Principal-Agent Problems, Econometrica 77(1), 249-278.

Conlon, John R. (2009b): Supplement to Two New Conditions Supporting the First-Order Approach to Multisignal Principal-Agent Problems, Econometrica Supplementary Material, 77, http://www.econometricsociety.org/ecta/Supmat/6688_proofs.pdf. 
Core, J. E. and W. R. Guay (2010): Is CEO Pay Too High and Are Incentives Too Low? A wealth-Based Contracting Framework. Academy of Management Perspectives 24(1), 5-19.

Core, J. E., W. R. Guay and D. F. Larcker (2003): Executive Equity Compensation and Incentives: a Survey. Federal Reserve Bank of New York Policy Review, 27-50.

Core, J. E., W. R. Guay and Randall S. Thomas (2005): Is U.S. CEO Compensation Inefficient Pay without Performance? Michigan Law Review 103(6), 1142-1185.

Dikolli, S., Diser, V. Hofmann, C. and T. Pfeiffer (2018): CEO Power and relative performance evaluation. Contemporary Accounting Research 35(3), 1279-1296.

Drymiotes, G. (2007): The monitoring role of insiders. Journal of Accounting and Economics 44 (3), 359-377.

Edmans, A. and Gabaix (2016): Executive Compensation. A Modern Primer, Journal of Economic Literature 54(4), 1232-1287.

Ferri, F. and R. F. Göx (2018): Executive Compensation, Corporate Governance, and Say on Pay, Foundations and Trends in Accounting 12(1), 1-103.

Frydman, C. and D. Jenter (2010), CEO Compensation, Annual Review of Financial Economics 2, 75-102.

Göx R. F. (2008): Tax Incentives for Inefficient Executive Pay and Reward for Luck, Review of Accounting Studies 13, 452-478.

Hemmer, T. (2004): Lessons Lost in Linearity: A Critical Assessment of the General Usefulness of LEN Models in Compensation Research, Journal of Management Accounting Research 16(1), 149-162.

Hemmer, T., Kim, O. and R. E. Verrecchia (2000): Introducing convexity into optimal compensation contracts, Journal of Accounting and Economics 28, 307-327.

Holmström, B. (1977): On Incentives and Control in Organizations. Ph.D. dissertation, Stanford University. 
Holmström, B. (1979): Moral Hazard and Observability, The Bell Journal of Economics, 10(1), 74-91.

Jensen M. and W. Meckling (1976): Theory of the firm: Managerial behavior, agency costs, and ownership structure. Journal of Financial Economics 3(4), 305-360

Jensen, M. C. and K. J. Murphy (1990): Performance pay and top management incentives, Journal of Political Economy 98, 225-265.

Jewitt, I. (1988): Justifying the first order approach to principal-agent problems, Econometrica 56, 1177-1190.

Kumar, P. and K. Sivaramakrishnan (2008): Who monitors the monitor? The effect of board independence on executive compensation and firm value. Review of Financial Studies 21 (3), 1371-1401.

Larcker, D. F. and Brian Tayan (2011): Corporate Governance Matters: A Closer Look at Organizational Choices and Their Consequences, FT Press.

Laux, V. and B. Mittendorf (2011): Board independence, executive pay, and the adoption of pet projects. Contemporary Accounting Research 28, 1467-1483.

Mirrlees, J. A. (1974): Notes on welfare economics, information and uncertainty. In Essays on Economic Behavior under Uncertainty. In: M. Balch, D. McFadden, and S-Y. Wu, eds. North Holland.

Mirrlees, J. A. (1999): The Theory of Moral Hazard and Unobservable Behaviour: Part I. Review of Economic Studies 66 (1), 3-21.

Murphy, K. (1999): Executive compensation. In: Ashenfelter, O. and D. Card., eds., Handbook of Labor Economics 3(B), 2485-2563, Elsevier.

Murphy, K. (2013): Executive Compensation: Where We Are, and How We Got There. In: Constantinides, G. M. Harris, M. and R. M. Stulz, eds, Handbook of the Economics of Finance 2(A), 211-356, Elsevier.

Tirole, J. (2006): The Theory of Corporate Finance. Princeton, NJ and Woodstock: Princeton University Press. 
Weisbach, M. 2007. Optimal Executive Compensation versus Managerial Power: A Review of Lucian Bebchuk and Jesse Fried's Pay without Performance: The Unfulfilled Promise of Executive Compensation. Journal of Economic Literature 45(2): 419-428. 
Figure 1: Board objective function for constrained and unconstrained solutions

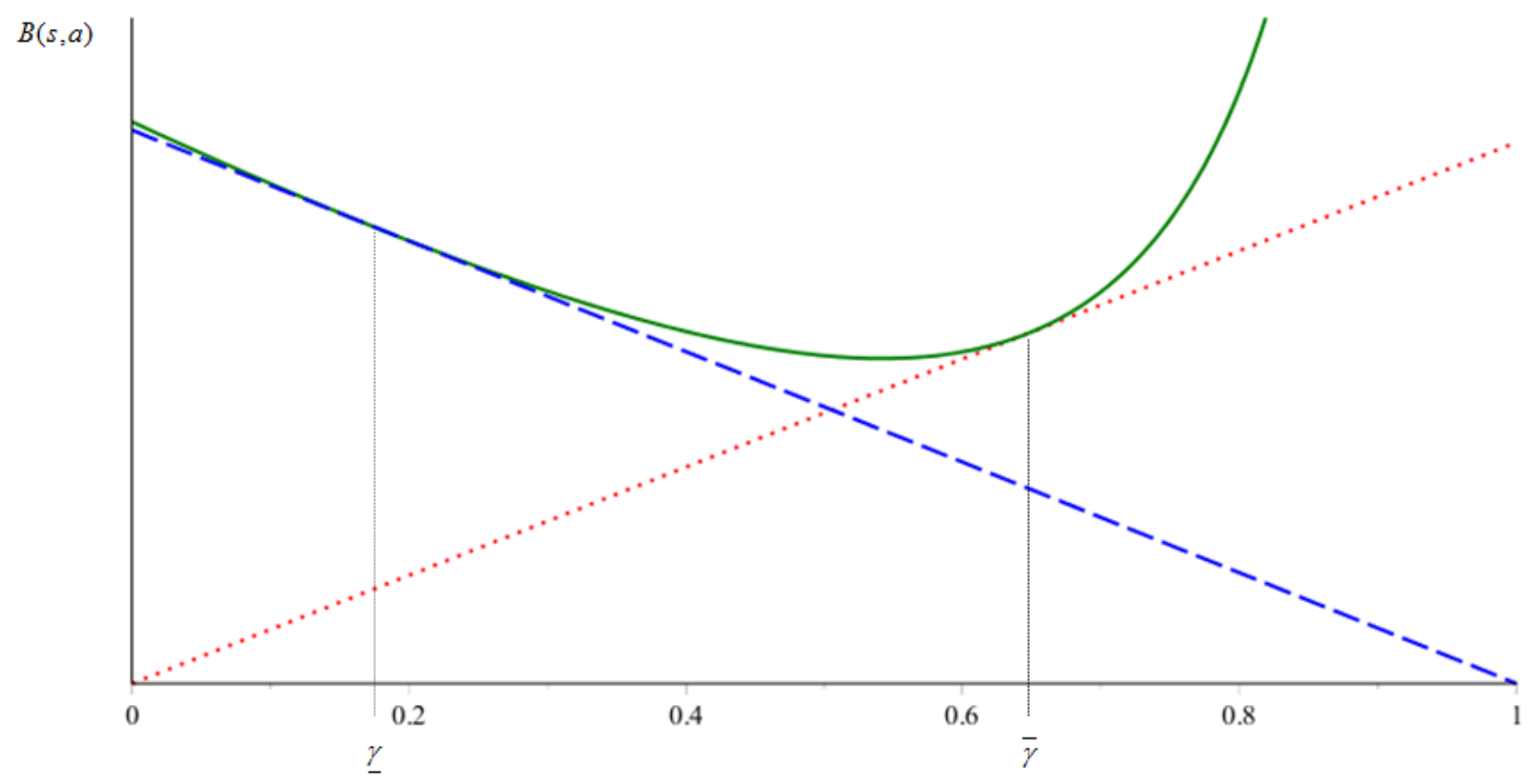

Figure 1 shows the board's objective function for the three solutions of the agency problem stated in Corollaries 1-3 as a function of the friendliness parameter $\gamma$. The dashed downward sloping line represents the board's expected utility for the standard contract in Corollary 1. This solution is optimal as long as $\gamma \leq \underline{\gamma}$. The dotted upward sloping line represents the board's expected utility for the contract with a binding participation constraint of shareholders in Corollary 2. This solution is optimal if $\gamma \geq \bar{\gamma}$. The solid convex curve shows the board's expected utility for the unconstrained solution in Corollary 3. This solution is optimal if This solution if $\gamma \in(\underline{\gamma}, \bar{\gamma})$. The plots were generated assuming that $H(s, a)=2 s^{1 / 2}-0.001 a$ and $x \sim \Gamma(0.015, a)$. 
Figure 2: Properties of optimal quadratic compensation contracts

\section{Panel A: Total compensation and pay-performance sensitivity with convex cost function}
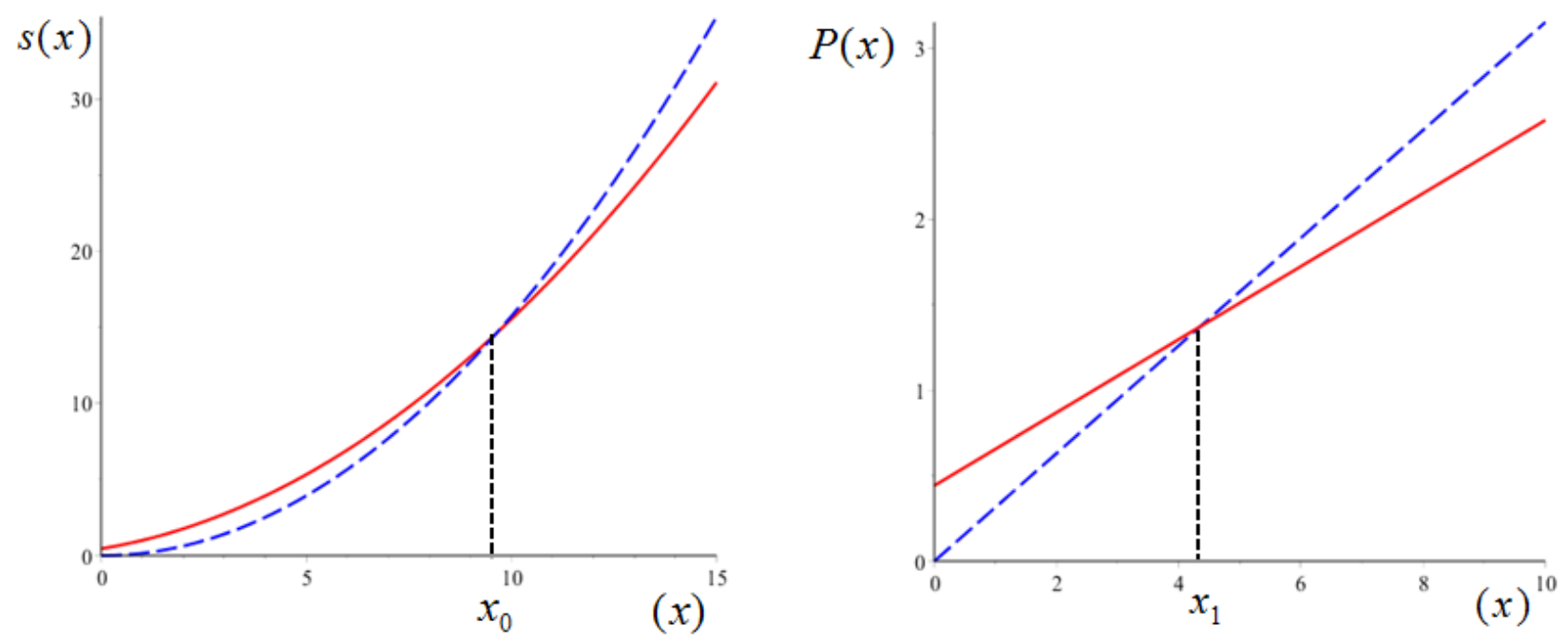

Panel B: Total compensation and pay-performance sensitivity with linear cost function
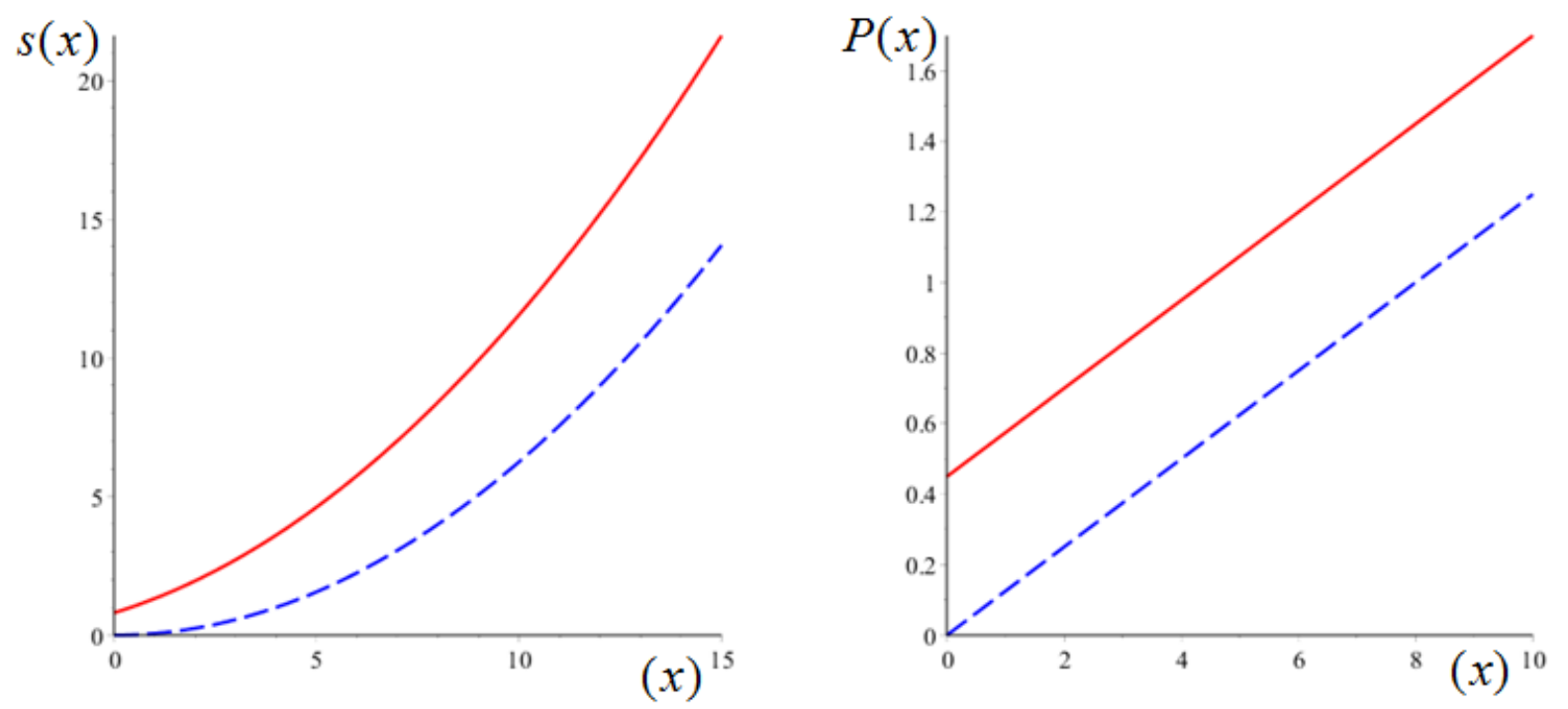

Figure 2 compares the essential properties of optimal quadratic compensation contracts for different degrees of board friendliness. Panel A shows the curvature of the optimal compensation function and the pay-performance sensitivity (PPS) for the cost function $C(a)=a^{2}$. Panel B shows the curvature of the optimal compensation function and the PPS for the cost function $C(a)=a$. In all figures, the dashed curves represent the standard contract proposed by a shareholder-friendly board $(\gamma \leq \underline{\gamma})$, whereas the solid curves represent the contract with a binding participation constraint of shareholders in Corollary 2 that is optimal for management-friendly boards with $\gamma \geq \bar{\gamma}$. The intersection points at $x=x_{0}\left(x=x_{1}\right)$ denote the critical performance levels above which the pay level (the PPS) of implemented by the CEO-friendly board is lower than the pay level (the PPS) implemented by the shareholder-friendly board. The plots were generated assuming a gamma distribution with a shape parameter of $k=2$. To assure that $z(x) \geq 0$ for all $x$, the plots in Panel A assume that $\underline{H}=C(a)$. 